\title{
Preventive antimicrobial action and tissue architecture ameliorations of Bacillus subtilis in challenged broilers
}

\author{
Essam S. Soliman'1(D), Rania T. Hamad² (i) and Mona S. Abdallah ${ }^{3}$
}

1. Department of Animal Hygiene, Zoonosis, and Animal Behavior, Faculty of Veterinary Medicine, Suez Canal University, Ismailia 41522, Egypt; 2. Department of Pathology, Faculty of Veterinary Medicine, Menoufia University, Al Minufya 33511, Egypt; 3. Department of Avian and Rabbit Medicine, Faculty of Veterinary Medicine, Suez Canal University, Ismailia 41522, Egypt.

Corresponding author: Essam S. Soliman, e-mail: soliman.essam@vet.suez.edu.eg

Co-authors: RTH: drrania_vet2007@yahoo.com, MSA: drmona_salim@yahoo.com

Received: 15-09-2020, Accepted: 19-01-2021, Published online: 26-02-2021

doi: www.doi.org/10.14202/vetworld.2021.523-536 How to cite this article: Soliman ES, Hamad RT, Abdallah MS (2021) Preventive antimicrobial action and tissue architecture ameliorations of Bacillus subtilis in challenged broilers, Veterinary World, 14(2): 523-536.

\begin{abstract}
Background and Aim: Probiotics improve intestinal balance through bacterial antagonism and competitive exclusion. This study aimed to investigate the in vitro antimicrobial activity, as well as the in vivo preventive, immunological, productive, and histopathological modifications produced by probiotic Bacillus subtilis.

Materials and Methods: The in vitro antimicrobial activities of B. subtilis $\left(5 \times 10^{6} \mathrm{CFU} / \mathrm{g} ; 0.5,1.0 *, 1.5\right.$, and $\left.2.0 \mathrm{~g} / \mathrm{L}\right) \mathrm{were}$ tested against Escherichia coli O157: H7, Salmonella Typhimurium, Candida albicans, and Trichophyton mentagrophytes after exposure times of $0.25,0.5,1$, and $2 \mathrm{~h}$ using minimal inhibitory concentration procedures. A total of 3201 -day-old female Ross broiler chickens were divided into five groups. Four out of the five groups were supplemented with 0.5 , $1.0^{*}$, 1.5 , and $2.0 \mathrm{~g} / \mathrm{L}$ probiotic B. subtilis from the age of 1 day old. Supplemented 14-day-old broiler chickens were challenged with only E. coli $\mathrm{O} 157$ : $\mathrm{H} 7\left(4.5 \times 10^{12} \mathrm{CFU} / \mathrm{mL}\right)$ and $S$. Typhimurium $\left(1.2 \times 10^{7} \mathrm{CFU} / \mathrm{mL}\right)$. A total of 2461 samples $(256$ microbial-probiotic mixtures, 315 sera, 315 duodenal swabs, and 1575 organs) were collected.

Results: The in vitro results revealed highly significant $(\mathrm{p}<0.001)$ killing rates at all-time points in $2.0 \mathrm{~g} / \mathrm{L}$ B. subtilis: 99.9\%, 90.0\%, 95.6\%, and 98.8\% against E. coli, S. Typhimurium, C. albicans, and T. mentagrophytes, respectively. Broilers supplemented with 1.5 and $2.0 \mathrm{~g} / \mathrm{L}$ B. subtilis revealed highly significant increases $(\mathrm{p}<0.01)$ in body weights, weight gains, carcass weights, edible organs' weights, immune organs' weights, biochemical profile, and immunoglobulin concentrations, as well as highly significant declines $(\mathrm{p}<0.01)$ in total bacterial, Enterobacteriaceae, and Salmonella counts. Histopathological photomicrographs revealed pronounced improvements and near-normal pictures of the livers and hearts of broilers with lymphoid hyperplasia in the bursa of Fabricius, thymus, and spleen after supplementation with $2.0 \mathrm{~g} / \mathrm{L}$ B. subtilis.
\end{abstract}

Conclusion: The studies revealed that $1.5-2.0 \mathrm{~g}$ of probiotic $B$. subtilis at a concentration of $5 \times 10^{6} \mathrm{CFU} / \mathrm{g} / \mathrm{L}$ water was able to improve performance, enhance immunity, and tissue architecture, and produce direct antimicrobial actions.

Keywords: broiler chickens, histopathological photomicrographs, immunity, in vitro antimicrobial, in vivo preventive, probiotics.

\section{Introduction}

Expansion in the poultry industry in the past 50 years has been accompanied by the emergence of a large variety of pathogens and increased microbial resistance. These effects have been attributed to the extensive and abnormal use of antibiotics as prophylactics and therapeutic agents. Recent research has focused on finding alternative supplements that minimize and/or prevent the maintenance of microbial agents in the environment, localization in target tissues, and the production of disease, as well as enhancing immunity levels in broiler chickens [1].

Copyright: Soliman, et al. Open Access. This article is distributed under the terms of the Creative Commons Attribution 4.0 International License (http://creativecommons.org/licenses/ by/4.0/), which permits unrestricted use, distribution, and reproduction in any medium, provided you give appropriate credit to the original author(s) and the source, provide a link to the Creative Commons license, and indicate if changes were made. The Creative Commons Public Domain Dedication waiver (http:// creativecommons.org/publicdomain/zero/1.0/) applies to the data made available in this article, unless otherwise stated.
Alternatives such as cytokines, bacteriophages [2], Nigella sativa Linn [3], cinnamon (Cinnamomum zeylanicum) oil [4], inorganic nano-selenium [5], and probiotics [6] have been suggested and found to be effective in improving performance and immunity.

Probiotics are living microorganisms that, when supplemented in adequate amounts and concentrations, result in health benefits [7]. Probiotic supplementation contributes to beneficial effects, such as improved performance, increased feed efficiency, improved nutrient digestion, and absorption [8], increased egg production, improved health, and reduced pathogenic enzyme secretion [9]. One study that investigated the expanded use of probiotics in poultry farming showed that newly hatched chickens from supplemented flocks could be protected against colonization of Salmonella Enteritidis with a dosing suspension of gut contents derived from healthy adult chickens [10], a concept is known as "competitive exclusion". 
In addition to competitive exclusion activity, probiotics exert their effects by regulating intestinal permeability, performing a foster action for the degradation and damage caused by enteric pathogens, enhancing humoral and cellular immunity, and altering agent pathogenicity [11]. Probiotics possess antimicrobial actions against many microorganisms, including Clostridium perfringens, Salmonella Typhimurium, Escherichia coli, and Staphylococcus aureus [12], by producing organic acids and antibacterial substances such as hydrogen peroxide, defensins, and bacteriocins.

This study aims to evaluate the in vitro antimicrobial action of probiotic Bacillus subtilis $\left(5 \times 10^{6}\right.$ $\mathrm{CFU} / \mathrm{g})$ at different concentrations $\left(0.5,1.0^{*}, 1.5\right.$, and $2.0 \mathrm{~g} / \mathrm{L})$ against $E$. coli $\mathrm{O} 157: \mathrm{H} 7\left(4.5 \times 10^{12} \mathrm{CFU} / \mathrm{mL}\right)$, $S$. Typhimurium $\left(1.2 \times 10^{7} \mathrm{CFU} / \mathrm{mL}\right)$, Candida albicans $\left(2.5 \times 10^{6} \mathrm{CFU} / \mathrm{mL}\right)$, and Trichophyton mentagrophytes $\left(2.5 \times 10^{6} \mathrm{CFU} / \mathrm{mL}\right)$ after different exposure intervals $(0.25,0.5,1$, and $2 \mathrm{~h})$ using minimal inhibitory concentration tests. The study also aims to study the in vivo preventive and prophylactic actions of probiotic $B$. subtilis on productive performance, histopathological picture, biochemical profile, intestinal microbial load, immune and edible organs' weights, and immunoglobulin ( $\mathrm{Ig}$ ) concentrations in 14-day-old broiler chickens challenged with $E$. coli $\mathrm{O} 157$ : $\mathrm{H} 7\left(4.5 \times 10^{12}\right.$ $\mathrm{CFU} / \mathrm{mL})$ and $S$. Typhimurium $\left(1.2 \times 10^{7} \mathrm{CFU} / \mathrm{mL}\right)$.

\section{Materials and Methods}

Ethical approval

The protocol and used materials of the current scientific research were approved by the Scientific Research Ethics Committee, Faculty of Veterinary Medicine, Suez Canal University, Ismailia, Egypt with approval number (2019004).

\section{Study period and location}

The in-vitro study was carried out during June and July 2019 in Animal, Poultry and Environmental Hygiene laboratories, Department of Animal Hygiene, Zoonosis and Animal Behavior and Management, Faculty of Veterinary Medicine, Suez Canal University, Ismailia. The in-vivo study was conducted from September $5^{\text {th }}, 2019$ to October $12^{\text {th }}, 2019$ in the Broiler Experimental Units, Faculty of Veterinary Medicine, Suez Canal University, Ismailia.

Carcasses' and organs' weights, bacteriological, and performance indices assessments were conducted in Animal, Poultry and Environmental Hygiene laboratories, Ismailia. Biochemical and immunoglobulin assays were conducted in the Clinical Pathology Laboratories, Suez Canal University Hospital. Histopathological examinations and photomicrography were conducted in the Pathology Department, Al Minufya.

\section{In vitro antimicrobial action of probiotics}

Preparation of probiotic suspensions

B. subtilis $\left(5 \times 10^{6} \mathrm{CFU} / \mathrm{g}\right)$ powder was purchased from a veterinary pharmacy in Ismailia, Egypt. The bag was opened with care, and four quantities were weighed $\left(0.5,1.0^{*}, 1.5\right.$, and $\left.2.0 \mathrm{~g}\right)$, and each weight was dissolved in $1 \mathrm{~L}$ of deionized water to produce the four targeted concentrations: $0.5,1.0 * 1.5$, and $2.0 \mathrm{~g} / \mathrm{L}$.

\section{Preparation of bacterial and fungal cultures}

E. coli $\mathrm{O} 157$ : $\mathrm{H} 7$ suspension $\left(1.8 \times 10^{7} \mathrm{CFU} / \mathrm{mL}\right)$ and $S$. Typhimurium lyophilized vials $\left(2.4 \times 10^{3} \mathrm{CFU}\right)$ were purchased from the Animal Health Research Institute, Dokki, Cairo. E. coli O157: H7 was propagated in MacConkey broth (Thermo Scientific ${ }^{\text {TM }}$ Oxoid $^{\mathrm{TM}}$ MacConkey Broth, CM0505, $500 \mathrm{~g}$ ) at $44^{\circ} \mathrm{C}$ for $24 \mathrm{~h}$, while $S$. Typhimurium was propagated in tetrathionate broth (Thermo Scientific ${ }^{\mathrm{TM}}$ Oxoid $^{\mathrm{TM}}$ Tetrathionate Broth Base, CM0029, 500 g) at $37^{\circ} \mathrm{C}$ for $24 \mathrm{~h}$, as recommended by Soliman et al. [13]. From the positive MacConkey and tetrathionate tubes, $10 \mu \mathrm{L}$ was dropped onto eosin methylene blue (EMB) agar (EMB, Modified Levine EMB Thermo Scientific ${ }^{\mathrm{TM}}$ Oxoid $^{\mathrm{TM}}$, CM0069B, 500 g) and CHROMagar ${ }^{\mathrm{TM}}$ (BD BBL ${ }^{\mathrm{TM}}$ CHROMagar ${ }^{\mathrm{TM}}$ Salmonella READY-TO-USE Plated Media) and

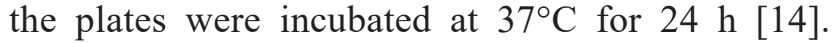
Metallic green colonies of E. coli O157: $\mathrm{H} 7$ and pink colonies of $S$. Typhimurium were counted, collected, and reconstituted in buffered peptone water (Thermo Scientific ${ }^{\mathrm{TM}}$ Oxoid $^{\mathrm{TM}}$ Buffered Peptone Water, CM0509B, $500 \mathrm{~g}$ ), providing suspensions of $4.5 \times 10^{12}$ and $1.2 \times 10^{7} \mathrm{CFU} / \mathrm{mL}$, respectively.

Suspensions of $C$. albicans and T. mentagrophytes $\left(3.5 \times 10^{3} \mathrm{CFU} / \mathrm{mL}\right)$ were provided by the Animal Health Research Institute of Ismailia. Fungal suspensions were propagated in Sabouraud Dextrose Broth (SDB, HIMEDIA ${ }^{\circledR}$ Sabouraud Dextrose Broth, MU033, $500 \mathrm{~g}$ ) at $37^{\circ} \mathrm{C}$ for $24 \mathrm{~h}$, dropped onto Sabouraud Dextrose agar (SDA, Thermo Scientific ${ }^{\mathrm{TM}}$

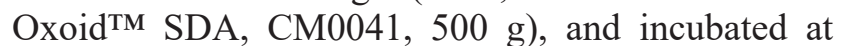
$37^{\circ} \mathrm{C}$ for $24 \mathrm{~h}$. Typical colonies were identified by morphological appearance and lactophenol cotton blue stain (Hardy Diagnostics ${ }^{\circledR}$ Lactophenol Cotton Blue, Z68, $15 \mathrm{~mL}$ ). Colonies were counted, collected, and reconstituted in SDB, providing suspensions of $2.5 \times 10^{6} \mathrm{CFU} / \mathrm{mL}$ for each organism.

\section{Testing probiotic concentrations against bacterial and fungal cultures}

The procedures were carried out using the minimal inhibitory concentration according to Soliman et al. [15]. One milliliter from each bacterial or fungal suspension was added to four replicates of $9 \mathrm{~mL}$ of each probiotic concentration $\left(0.5,1.0^{*}, 1.5\right.$, and $2.0 \mathrm{~g} / \mathrm{L}$ ), and mixed using a vortexer (Vortex Mixer XH-D, $2800 \mathrm{r} / \mathrm{m}, 30 \mathrm{~W}$, bowel and disk shapes). After $0.25,0.5,1$, and $2 \mathrm{~h}$ of exposure time, $100 \mu \mathrm{L}$ were transferred and added to $10-\mathrm{mL}$ physiological saline resuscitation tubes held previously at $4{ }^{\circ} \mathrm{C}$ and mixed thoroughly by vortexing. The tubes were transferred for the bacteriological assessment. 


\section{In vivo efficiency of probiotics}

Experimental birds: Microclimate and management

A total of 320 1-day-old female Ross chicks were purchased from a company in Ismailia, Egypt. Broiler chicks were divided on their arrival into five groups: G1, G2, G3, G4, and G5 (control), 64 chicks in each group (four replicates of 16 birds). Groups were placed into five independent rooms. The floors of the five rooms were treated with superphosphate $\left(0.5 \mathrm{~g} / \mathrm{m}^{2}\right)$ before being covered with a hay deep litter system according to Soliman and Hassan [16]. Each room was ventilated through fans on one sidewall and V-shaped windows on the opposing sidewall, contributing to negative pressure cross-ventilation across the room. Broiler chicks in each room were supplied with a continuous lighting regimen using blue LED lights that were adjusted to provide $23 \mathrm{~h}$ of lighting and $1 \mathrm{~h}$ of darkness a day, as recommended by Soliman and Hassan [17]. The five rooms were secured using the essential biosecurity measures recommended by Soliman and Abdallah [18]: Fly-proof nets, foot dips at the entrance, controlled traffic in and out, restricted access to the rooms, protection of the food storage areas, protection of water resources, and protection from wild bird entrance.

Before the broilers' arrival, the microclimatic temperature was adjusted and maintained in the five rooms at $35^{\circ} \mathrm{C}$ (brooding temperature) using halogen heaters (Bravo BR-4T Heater 4 halogen Candles, $2400 \mathrm{~W})$. The indoor temperature was controlled and minimized at a rate of $3^{\circ} \mathrm{C} /$ week by increasing the ventilation rates during the daylight hours until $26^{\circ} \mathrm{C}$ was achieved by the end of the $3^{\text {rd }}$ week. Birds were given ad libitum access to dechlorinated water and were supplied with a corn-soybean ration to meet their nutritional requirements, as recommended by the National Research Council [19] and Applegate and Angel [20]. Broilers were provided with the corn-soybean ration into two successive stages: The starter ration was provided from 1 to 13 days and constituted $23 \%$ protein, $3.81 \%$ crude fiber, $4.9 \%$ fat, and $2950 \mathrm{kcal} / \mathrm{kg}$ energy; and the grower ration was provided from 14 days until the end of the fattening cycle (38 days) and constituted $21 \%$ protein, $3.39 \%$ crude fiber, $5.8 \%$ fat, and $3100 \mathrm{kcal} / \mathrm{kg}$ energy. The experiment was designed to last for 38 days. Survival rates, microclimatic thermal level, and humidity level were monitored during the experiment.

Broilers were immunized by mass vaccination in dechlorinated drinking water during the early morning after water deprivation for 2-3 h. Birds were vaccinated against infectious bronchitis using PESTIKAL B1 SPF H120 $\geq 10^{3.5}$ live attenuated virus vaccine on the $6^{\text {th }}$ day, against infectious bursal disease using SERVAC D78 Strain VMG91 $\geq 10^{3.0}$ live attenuated vaccine on the $14^{\text {th }}$ and $21^{\text {st }}$ days, and against Newcastle disease (ND) virus using PESTIKAL LaSota $\geq 10^{6.0}$ live lentogenic ND virus on the $18^{\text {th }}$ and $28^{\text {th }}$ days.

\section{Probiotic supplementation}

The chicks in four out of the five broiler groups (G1, G2, G3, and G4) were given drinking water supplemented with $B$. subtilis $\left(5 \times 10^{6} \mathrm{CFU} / \mathrm{g}\right)$ from 1 day of age at a rate of $0.5,1.0 *$ (recommended by the manufacturer), 1.5 , and $2.0 \mathrm{~g} / \mathrm{L}$, respectively. The fifth group was used as an unsupplemented control group.

\section{E. coli O157: H7 and S. Typhimurium challenge}

Broilers in groups G1, G2, G3, and G4 were challenged with $E$. coli $\mathrm{O} 157$ : $\mathrm{H} 7\left(4.5 \times 10^{12} \mathrm{CFU} / \mathrm{mL}\right)$ and $S$. Typhimurium $\left(1.2 \times 10^{7} \mathrm{CFU} / \mathrm{mL}\right)$ in the drinking water at 14 days of age [21].

\section{Performance indices}

The live body weights of the broiler groups were measured by weighing 56 birds from each group. The number of the weighed birds was calculated using the simple random sampling procedures described by Thrusfield [22] with a 5\% error as following:

$$
n=1.96^{2} P_{\text {exp }}\left(1-P_{\text {exp }}\right) / d^{2}
$$

Where $n=$ required sample size, Pexp=expected prevalence, $d=$ desired absolute precision. Feed intakes expressed by grams (g) were calculated in each group by dividing the total amount consumed by the birds in such a group by the total number of surviving birds in the group. Weight gains expressed by $(\mathrm{g})$, feed conversion ratios (FCR), and performance indices were calculated as recommended by Soliman and Hassan [23].

\section{Sampling}

A total of 2461 samples, including 256 in vitro microbial-probiotic mixtures, 315 sera, 315 duodenal swabs, and 1,575 organs (including bursa of Fabricius, spleen, thymus, heart, and liver) were collected during the period of the study.

Blood samples (a total of 315 sera samples) were obtained by sacrificing 63 birds in each of the five groups by the end of the study (38 days). The blood samples were received in sterile screw-capped centrifuge tubes, held at $37^{\circ} \mathrm{C}$ for $30 \mathrm{~min}$, and centrifuged (Fisher ${ }^{\circledR}$ Thermo Scientific CL10 Centrifuge with F-G3 Rotor, max rpm: 4000) at $3000 \mathrm{rpm}$ for 15 min. Clear non-hemolyzed sera samples were pipetted using an automatic pipette (Thermo Scientific ${ }^{\mathrm{TM}}$ Finnpipette $^{\mathrm{TM}}$ Adjustable Volume Single-Channel Micro Pipettor, 100-1000 $\mu \mathrm{L}$ volume) into 2-mL Eppendorf tubes and stored at $-20^{\circ} \mathrm{C}$ for the biochemical and immunological assays.

Duodenal swabs (315 swabs and 63 per group) were collected from the intestines of sacrificed broilers' and placed in $9 \mathrm{~mL}$ of buffered peptone water (Thermo Scientific ${ }^{\mathrm{TM}}$ Oxoid $^{\mathrm{TM}}$ Buffered Peptone Water, CM0509B, $500 \mathrm{~g}$ ); and the in vitro microbial-probiotic mixtures (256 samples, 4 replicates $\times 4$ contact times $\times 4$ cultures $\times 4$ probiotic concentrations) in the physiological saline resuscitation tubes were transferred for bacteriological assessment. 
A total of 315 birds were slaughtered after blood sampling and decapitation; the shanks and feet were removed with a knife, and birds were de-feathered and eviscerated of all organs except kidneys. The carcasses were weighed and expressed in grams (carcass weight: $\mathrm{CW} / \mathrm{g}$ ). Edible organs such as the heart and liver, and the immune organs (bursa of Fabricius, spleen, and thymus) were removed, weighed, and recorded as $\mathrm{g} / \mathrm{kg}$ bodyweight. All organs were kept in $10 \%$ formalin for histopathological examination. Sacrificed birds were hygienically disposed of after sampling through burial of the carcasses, heads, shanks, feet, and viscera with the use of slaked lime beneath and above, and the area was fenced to discourage carnivorous animals.

\section{Biochemical and immunological assay}

Sera (a total of 315 sera samples were collected from 315 sacrificed birds: 63 birds from each experimented group) were examined for levels of total protein (expressed in $\mathrm{g} / \mathrm{dL}$ ), albumin (expressed in $\mathrm{g} / \mathrm{dL}$ ), alanine aminotransferase (expressed in IU/L), aspartate aminotransferase (expressed in IU/L), urea (expressed in $\mathrm{mg} / \mathrm{dL}$ ), and creatinine (expressed in $\mathrm{mg} / \mathrm{dL}$ ) using a Roche COBAS Integra 800 Chemistry Analyzer. Ig (IgG, IgM, and IgA; expressed in $\mathrm{mg} / \mathrm{dL}$ ) concentrations were measured using a Roche Elecsys 1010 Immunoassay Analyzer.

\section{Bacteriological examination}

Ten microliters from each of the in vitro microbial-probiotic mixture $(256$ samples, 4 replicates $\times 4$ contact times $\times 4$ cultures $\times 4$ probiotics concentrations) resuscitation tubes were dropped (using the drop plate technique as recommended by Kim and Lee [24]) onto EMB agar (Modified Levine EMB Thermo Scientific ${ }^{\mathrm{TM}}$ Oxoid $^{\mathrm{TM}}$, CM0069B, $500 \mathrm{~g}$ ) and CHROMagar (BD BBL ${ }^{\mathrm{TM}}$ CHROMagar ${ }^{\mathrm{TM}}$ Salmonella READY-TO-USE Plated Media) for the E. coli $\mathrm{O} 157$ : $\mathrm{H} 7$ and $S$. Typhimurium assays, respectively, and onto SDA (Thermo Scientific ${ }^{\mathrm{TM}}$ Oxoid $^{\mathrm{TM}}$ SDA, CM0041, $500 \mathrm{~g}$ ) for the C. albicans and T. mentagrophytes assays and incubated at $37^{\circ} \mathrm{C}$ for $24 \mathrm{~h}$.

Duodenal swabs (a total of 315 duodenal swabs were collected after sacrificing 315 birds: 63 birds from each experimented group) were prepared according to the method described by the American Public Health Association $[25,26]$. In brief, ten-fold serial dilutions up to $10^{-8}$ were prepared to screen the different opportunities for microbial growth. Bacterial counts were conducted using the drop plate technique, as recommended by Kim and Lee [24], onto standard plate count agar (SPA, Thermo Scientific ${ }^{\mathrm{TM}}$ Oxoid $^{\mathrm{TM}}$ Plate Count Agar, CM0325, 500 g), EMB agar (Modified Levine Eosine Methylene Blue Thermo Scientific ${ }^{\mathrm{TM}}$ Oxoid $^{\mathrm{TM}}$, CM0069B, $500 \mathrm{~g}$ ), and CHROMagar (BD

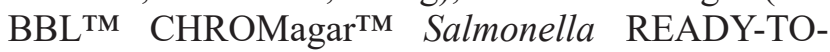
USE Plated Media) for the total aerobic bacterial count, total Enterobacteriaceae count (TEC), and total Salmonella count (TSC), and the plates were incubated at $37^{\circ} \mathrm{C}$ for $24-48 \mathrm{~h}$.

Typical colonies of E. coli, Salmonella, Candidal Trichophyton on EMB agar, CHROMagar, and SDA, respectively, during the in vitro study were detected, counted, and compared to the original microbial counts used, and the killing rates were calculated. Total bacterial count on SPA, TEC on EMB agar, and TSC on CHROMagar cultured from the in vivo intestinal swabs that showed 30-300 CFU were recorded. The counting of microbial colonies during the in vitro and in vivo studies was carried using a darkfield colony counter (R164109 Reichert-Jung Quebec Darkfield 3325 Colony Counter, Fisher Scientific) [27], and the counts were converted into logarithmic numbers.

\section{Histopathological examination}

Tissue samples from the liver, heart, spleen, thymus, and bursa of Fabricius were washed with 5\% phosphate-buffered saline (PBS) (ABI ${ }^{\circledR} \mathrm{PBS}, \mathrm{PBS}, 10 \times$ concentrated, sterile, $\mathrm{pH} 7.4$, without $\mathrm{CaCl}_{2}$ and $\mathrm{MgCl}_{2}$, $500 \mathrm{~mL}$ ) and fixed through impregnation in $10 \%$ buffered formalin saline solution. The tissues were maintained until complete fixation, cut into sections of 5-mm thickness, and put into tissue cassettes. The specimens were dehydrated through transfer through a series of alcohols with different concentrations, cleared with two changes of xylol, embedded into paraffin, and cut into $4-\mu \mathrm{m}$-thick sections. The obtained tissue sections were stained with hematoxylin and eosin as recommended by Bancroft et al. [28] and Jones et al. [29]. Histological sections were examined using a Zeiss Axioplan microscope (Carl Zeiss MicroImaging, Thornwood, NY, USA) under $40 \times$ and photographed.

\section{Statistical analysis}

Statistical analysis was carried out using SPSS version 20 (SPSS-20, IBM Corp., NY, USA) [30]. The recorded data were analyzed using a two-way multifactorial analysis of variance for all treated groups, age, and their interactions. Data were analyzed for the in vitro influence of different probiotic concentrations $\left(0.5,1.0^{*}, 1.5\right.$, and $\left.2.0 \mathrm{~g} / \mathrm{L}\right)$ on microbial cultures after different exposure intervals $(0.25,0.5,1$, and $2 \mathrm{~h})$, and for the in vivo influence of the probiotic concentration, broiler age, and their interactions on performance, immunity, carcass weight, immune organ weight, edible organ weights, and intestinal microbial counts. The statistical model was summarized as follow:

$$
\mathrm{Y}_{\mathrm{ijk}}=\mu+\alpha_{\mathrm{i}}+\beta_{\mathrm{j}}+(\alpha \beta)_{\mathrm{ij}}+\varepsilon_{\mathrm{ijk}}
$$

Where $Y_{\mathrm{ijk}}$ was the measurement of the dependent variables; $\mu$ was overall mean; $\alpha_{i}$ was the fixed effect of the probiotic concentrations; $\beta_{\mathrm{j}}$ was the fixed effect of the broiler's age; $(\alpha \beta)_{i j}$ was the interaction effect of the probiotic concentrations by broiler's age; and $\varepsilon_{\mathrm{ijk}}$ was the random error. The results were displayed in tables as high significance at $\mathrm{p}<0.01$, significant at $\mathrm{p} \leq 0.05$, and non-significant at $\mathrm{p}>0.05$. The logarithmic forms $\left(\log _{10}\right)$ of total bacterial, Enterobacteriaceae, and 
Salmonella counts were calculated using Microsoft Excel 2016.

\section{Results}

\section{In vitro antimicrobial actions}

The in vitro antimicrobial actions are shown in Table-1. The overall in vitro antimicrobial actions of the probiotic revealed highly significant reductions $(\mathrm{p}<0.01)$ in E. coli O157: H7, S. Typhimurium, C. albicans, and T. mentagrophytes viable counts (up to $99.9 \%, 55.7 \%, 55.1 \%$, and $60.2 \%$, respectively) when exposed to $2.0 \mathrm{~g} / \mathrm{L} \mathrm{B}$. subtilis suspension compared to other concentrations, regardless of the contact time. The probiotic revealed highly significant antimicrobial actions $(p<0.01)$ as the contact time with the microbial cultures increased to $2 \mathrm{~h}$.

Different contact times conferred highly significant reductions $(\mathrm{p}<0.01)$ in the E. coli O157: H7 viable count: up to $99.8 \%$ in $0.5 \mathrm{~g} / \mathrm{L}$ B. subtilis suspension after $1 \mathrm{~h}, 100.0 \%$ in $1.0^{*} \mathrm{~g} / \mathrm{L}$ B. subtilis suspension after $1 \mathrm{~h}, 100.0 \%$ in $1.5 \mathrm{~g} / \mathrm{L} B$. subtilis suspension after $1 \mathrm{~h}$, and $100.0 \%$ in $2.0 \mathrm{~g} / \mathrm{L} \mathrm{B}$. subtilis suspension after $0.5 \mathrm{~h}$. No significant differences were recorded between the killing efficacies recorded as a result of the exposure to 1.5 or $2.0 \mathrm{~g} / \mathrm{L} \mathrm{B}$. subtilis suspension. $S$. Typhimurium also showed highly significant reductions $(\mathrm{p}<0.01)$ : up to $68.5 \%$ in 0.5 $\mathrm{g} / \mathrm{L} B$. subtilis suspension after $2 \mathrm{~h}, 74.2 \%$ in $1.0^{*}$ $\mathrm{g} / \mathrm{L}$ B. subtilis suspension after $2 \mathrm{~h}, 84.2 \%$ in $1.5 \mathrm{~g} / \mathrm{L}$ B. subtilis suspension after $2 \mathrm{~h}$, and $90.0 \%$ in $2.0 \mathrm{~g} / \mathrm{L}$ B. subtilis suspension after $2 \mathrm{~h}$.

The yeast assays also revealed significant viable cell count reduction rates. C. albicans showed highly significant reductions $(\mathrm{p}<0.01)$ : $\mathrm{Up}$ to $91.0 \%$ in $0.5 \mathrm{~g} / \mathrm{L}$ B. subtilis suspension after $2 \mathrm{~h}, 94.2 \%$ in $1.0^{*} \mathrm{~g} / \mathrm{L}$ B. subtilis suspension after $2 \mathrm{~h}, 94.8 \%$ in $1.5 \mathrm{~g} / \mathrm{L}$ B. subtilis suspension after $2 \mathrm{~h}$, and $95.6 \%$ in $2.0 \mathrm{~g} / \mathrm{L} \mathrm{B}$. subtilis suspension after 2 h. T. mentagrophytes also showed highly significant reductions

Table-1: In vitro antimicrobial action (killing rate mean \pm SE) of different Bacillus subtilis probiotic' concentrations at different exposure times.

\begin{tabular}{|c|c|c|c|c|c|}
\hline \multirow[t]{2}{*}{ Probiotic g/L } & \multirow[t]{2}{*}{ Contact times/h } & \multicolumn{2}{|c|}{ Bacterial cultures (\%) } & \multicolumn{2}{|c|}{ Fungal cultures (\%) } \\
\hline & & $\begin{array}{c}\text { Escherichia coli } \\
\text { 0157: H7 }\end{array}$ & $\begin{array}{c}\text { Salmonella } \\
\text { Typhimurium }\end{array}$ & $\begin{array}{l}\text { Candida } \\
\text { albicans }\end{array}$ & $\begin{array}{c}\text { Trichophyton } \\
\text { mentagrophytes }\end{array}$ \\
\hline \multicolumn{6}{|c|}{ Overall means concerning probiotic concentrations } \\
\hline $0.5 \mathrm{~g} / \mathrm{L}$ & & $84.3^{\mathrm{a}} \pm 0.61$ & $34.2^{\mathrm{d}} \pm 0.15$ & $52.2^{\mathrm{a}} \pm 0.07$ & $56.2^{\mathrm{b}} \pm 0.8$ \\
\hline $1.0 * \mathrm{~g} / \mathrm{L}$ & & $99.2^{\mathrm{a}} \pm 0.33$ & $40.0^{c} \pm 0.26$ & $52.6^{\mathrm{a}} \pm 0.08$ & $57.0^{\mathrm{a}, \mathrm{b}} \pm 0.07$ \\
\hline $1.5 \mathrm{~g} / \mathrm{L}$ & & $99.9^{a} \pm 0.01$ & $50.0^{\mathrm{b}} \pm 0.33$ & $54.0^{\mathrm{a}} \pm 0.07$ & $59.6^{\mathrm{a}, \mathrm{b}} \pm 0.09$ \\
\hline $2.0 \mathrm{~g} / \mathrm{L}$ & & $99.9^{\mathrm{a}} \pm 0.00$ & $55.7^{\mathrm{a}} \pm 0.16$ & $55.1^{\mathrm{a}} \pm 0.09$ & $60.2^{\mathrm{a}} \pm 0.06$ \\
\hline p-value & & 0.001 & 0.000 & 0.334 & 0.069 \\
\hline \multicolumn{6}{|c|}{ Overall means concerning contact times } \\
\hline $0.25 \mathrm{~h}$ & & $84.3^{b} \pm 0.31$ & $18.5^{\mathrm{d}} \pm 0.18$ & $13.2^{\mathrm{d}} \pm 0.08$ & $18.0^{\mathrm{d}} \pm 0.05$ \\
\hline $0.5 \mathrm{~h}$ & & $99.2^{\mathrm{a}} \pm 0.25$ & $27.8^{\complement} \pm 0.19$ & $35.2^{\mathrm{c}} \pm 1.19$ & $40.0^{\circ} \pm 1.23$ \\
\hline $1 \mathrm{~h}$ & & $99.9^{\mathrm{a}} \pm 0.01$ & $54.2^{b} \pm 0.36$ & $73.2^{\mathrm{b}} \pm 1.65$ & $78.0^{\mathrm{b}} \pm 0.69$ \\
\hline $2 \mathrm{~h}$ & & $100.0^{\mathrm{a}} \pm 0.01$ & $79.2^{\mathrm{a}} \pm 2.22$ & $92.2^{\mathrm{a}} \pm 0.53$ & $97.0^{\mathrm{a}} \pm 0.61$ \\
\hline p-value & & 0.005 & 0.001 & 0.000 & 0.002 \\
\hline \multicolumn{6}{|c|}{ Probiotics concentrations by contact times interactions } \\
\hline \multirow[t]{4}{*}{$0.5 \mathrm{~g} / \mathrm{L}$} & $0.25 \mathrm{~h}$ & $40.4^{c} \pm 4.12$ & $7.8^{\mathrm{d}} \pm 0.71$ & $12.0 \pm 1.63$ & $16.0^{\mathrm{d}} \pm 1.55$ \\
\hline & $0.5 \mathrm{~h}$ & $97.0^{\mathrm{b}} \pm 0.20$ & $17.1^{\mathrm{c}} \pm 0.00$ & $34.0^{c} \pm 2.58$ & $38.0^{c} \pm 1.32$ \\
\hline & $1 \mathrm{~h}$ & $99.8^{\mathrm{a}} \pm 0.01$ & $43.5^{\mathrm{b}} \pm 2.14$ & $72.0^{\mathrm{b}} \pm 0.65$ & $76.0^{\mathrm{b}} \pm 2.11$ \\
\hline & $2 \mathrm{~h}$ & $99.9^{\mathrm{a}} \pm 0.00$ & $68.5^{\mathrm{a}} \pm 1.16$ & $91.0^{\mathrm{a}} \pm 1.01$ & $95.0^{\mathrm{a}} \pm 1.02$ \\
\hline \multirow[t]{4}{*}{$1.0 * \mathrm{~g} / \mathrm{L}$} & $0.25 \mathrm{~h}$ & $97.0^{\mathrm{b}} \pm 0.20$ & $13.5^{d} \pm 0.66$ & $12.4^{\mathrm{d}} \pm 1.22$ & $16.8^{\mathrm{d}} \pm 0.98$ \\
\hline & $0.5 \mathrm{~h}$ & $99.8^{\mathrm{a}} \pm 0.01$ & $22.8^{c} \pm 0.00$ & $38.1^{c} \pm 0.99$ & $39.2^{c} \pm 0.56$ \\
\hline & $1 \mathrm{~h}$ & $100.0^{a} \pm 0.00$ & $49.2^{b} \pm 1.98$ & $75.8^{\mathrm{b}} \pm 3.61$ & $77.4^{\mathrm{b}} \pm 1.12$ \\
\hline & $2 \mathrm{~h}$ & $100.0^{a} \pm 0.00$ & $74.2^{\mathrm{a}} \pm 1.22$ & $94.2^{\mathrm{a}} \pm 1.55$ & $95.3^{a} \pm 0.01$ \\
\hline \multirow[t]{4}{*}{$1.5 \mathrm{~g} / \mathrm{L}$} & $0.25 \mathrm{~h}$ & $99.8^{\mathrm{a}} \pm 0.01$ & $23.5^{d} \pm 0.82$ & $13.8^{d} \pm 1.55$ & $19.4^{d} \pm 0.02$ \\
\hline & $0.5 \mathrm{~h}$ & $99.9^{a} \pm 0.00$ & $32.8^{c} \pm 0.00$ & $39.8^{c} \pm 2.58$ & $41.4^{c} \pm 0.52$ \\
\hline & $1 \mathrm{~h}$ & $100.0^{a} \pm 0.00$ & $59.2^{b} \pm 0.98$ & $75.8^{\mathrm{b}} \pm 3.66$ & $79.2^{\mathrm{b}} \pm 1.32$ \\
\hline & $2 \mathrm{~h}$ & $100.0^{a} \pm 0.00$ & $84.2^{\mathrm{a}} \pm 1.32$ & $94.8^{\mathrm{a}} \pm 1.21$ & $98.1^{\mathrm{a}} \pm 1.08$ \\
\hline \multirow[t]{4}{*}{$2.0 \mathrm{~g} / \mathrm{L}$} & $0.25 \mathrm{~h}$ & $99.9^{\mathrm{a}} \pm 0.00$ & $29.2^{d} \pm 1.66$ & $14.8^{d} \pm 1.66$ & $20.1^{d} \pm 0.62$ \\
\hline & $0.5 \mathrm{~h}$ & $100.0^{a} \pm 0.00$ & $38.5^{\complement} \pm 0.62$ & $41.5^{\complement} \pm 2.55$ & $42.0^{c} \pm 1.54$ \\
\hline & $1 \mathrm{~h}$ & $100.0^{a} \pm 0.00$ & $65.0^{\mathrm{b}} \pm 1.54$ & $79.4^{\mathrm{b}} \pm 1.15$ & $80.0^{\mathrm{b}} \pm 1.54$ \\
\hline & $2 \mathrm{~h}$ & $100.0 \pm 0.00$ & $90.0^{\mathrm{a}} \pm 0.02$ & $95.6^{a} \pm 0.98$ & $98.8^{\mathrm{a}} \pm 1.00$ \\
\hline p-value & & 0.094 & 0.002 & 0.001 & 0.000 \\
\hline
\end{tabular}

Means carrying different superscripts in the same column are significantly different at $\mathrm{P} \leq 0.05$ or highly significantly different at $\mathrm{P}<0.01$. Means carrying the same superscripts in the same column are non-significantly different at $\mathrm{P}<0.05$. $\mathrm{SE}=$ Standard error, $*$ Recommended concentration by the manufacturer 
( $\mathrm{p}<0.01$ ): Up to $95.0 \%$ in $0.5 \mathrm{~g} / \mathrm{L}$ B. subtilis solution after $2 \mathrm{~h}, 95.3 \%$ in $1.0^{*} \mathrm{~g} / \mathrm{L}$ B. subtilis solution after $2 \mathrm{~h}, 98.1 \%$ in $1.5 \mathrm{~g} / \mathrm{L}$ B. subtilis suspension after $2 \mathrm{~h}$, and $98.8 \%$ in $0.5 \mathrm{~g} / \mathrm{L}$ B. subtilis suspension after $2 \mathrm{~h}$.

\section{Growth traits}

The effects of different concentrations of B. subtilis suspension on the growth traits of broilers are shown in Table-2. The monitoring and observations of the broilers that received probiotic treatment revealed significantly lower mortalities: $1.3 \%$ (3 out of 230 broilers) during the entire experiment. Weight gains and performance indices revealed highly significant increases $(\mathrm{p}<0.01)$ in broilers supplemented with $2.0,1.5,1.0^{*}$, and $0.5 \mathrm{~g} / \mathrm{L} B$. subtilis suspension (Table-2). Feed intakes showed highly significant declines $(\mathrm{p}<0.01)$ in broilers supplemented with $2.0,1.5,1.0^{*}$, and $0.5 \mathrm{~g} / \mathrm{L} B$. subtilis suspension.
FCR revealed highly significant $(\mathrm{p}<0.01)$ lower and promising ratios in broilers supplemented with 2.0, 1.5, 1.0*, and $0.5 \mathrm{~g} / \mathrm{L}$ B. subtilis suspension. Age interactions with the different treatments showed highly significant increases $(\mathrm{p}<0.01)$ in weight gains and performance indices at the $3^{\text {rd }}, 5^{\text {th }}, 4^{\text {th }}, 2^{\text {nd }}$, and $1^{\text {st }}$ weeks, respectively, and highly significant increases $(p<0.01)$ in feed intakes as age proceeded. Highly significant increases $(p<0.01)$ were also observed in the FCR at the $5^{\text {th }}, 4^{\text {th }}, 3^{\text {rd }}, 1^{\text {st }}$, and $2^{\text {nd }}$ weeks, respectively.

\section{Live, carcass, and immune organ weights}

The effects of different concentrations of $B$. subtilis suspension on the live, carcass, and immune organ weights of broilers are shown in Table-3. Live body, carcasses, immune organs (bursa of Fabricius and thymus), and edible organs (liver and heart) weights,

Table-2: Performance indices (mean \pm SE) in broilers supplemented with different concentrations of probiotics.

\begin{tabular}{|c|c|c|c|c|c|}
\hline Probiotic g/L & Age/week & BWG/g & $\mathbf{F I} / \mathbf{g}$ & FCR & PI \\
\hline \multicolumn{6}{|c|}{ Overall means concerning probiotic concentrations } \\
\hline $0.5 \mathrm{~g} / \mathrm{L}$ & & $364.6^{c, d} \pm 6.62$ & $580.7^{b} \pm 5.95$ & $1.58^{\mathrm{a}} \pm 0.13$ & $6.24^{\mathrm{d}} \pm 0.53$ \\
\hline $1.0 * \mathrm{~g} / \mathrm{L}$ & & $383.2^{b, c} \pm 2.95$ & $563.2^{c} \pm 5.84$ & $1.47^{\mathrm{b}} \pm 0.11$ & $6.94^{c} \pm 0.62$ \\
\hline $1.5 \mathrm{~g} / \mathrm{L}$ & & $400.8^{b} \pm 3.12$ & $526.0^{d} \pm 5.36$ & $1.33^{c} \pm 0.11$ & $8.24^{\mathrm{b}} \pm 0.72$ \\
\hline $2.0 \mathrm{~g} / \mathrm{L}$ & & $421.9^{a} \pm 32.4$ & $504.0^{e} \pm 5.29$ & $1.17^{\mathrm{d}} \pm 0.10$ & $9.23^{a} \pm 0.82$ \\
\hline Control & & $349.0^{\mathrm{d}} \pm 2.38$ & $589.1^{\mathrm{a}} \pm 5.95$ & $1.66^{\mathrm{a}} \pm 0.13$ & $5.71^{\mathrm{e}} \pm 0.45$ \\
\hline p-value & & 0.000 & 0.001 & 0.000 & 0.000 \\
\hline \multicolumn{6}{|c|}{ Overall means concerning contact times } \\
\hline $1^{\text {st }}$ week & & $116.0^{\mathrm{d}} \pm 1.84$ & $129.1^{\mathrm{e}} \pm 2.39$ & $1.12^{\mathrm{c}} \pm 0.02$ & $1.35^{\mathrm{e}} \pm 0.05$ \\
\hline $2^{\text {nd }}$ week & & $403.1^{\mathrm{c}} \pm 4.16$ & $299.8^{\mathrm{d}} \pm 4.79$ & $0.74^{\mathrm{d}} \pm 0.01$ & $7.49^{d} \pm 0.18$ \\
\hline $3^{\text {rd }}$ week & & $537.8^{\mathrm{a}} \pm 7.74$ & $590.4^{c} \pm 5.44$ & $1.10^{c} \pm 0.02$ & $10.02^{a} \pm 0.29$ \\
\hline $4^{\text {th }}$ week & & $418.0^{c} \pm 2.26$ & $773.8^{\mathrm{b}} \pm 5.92$ & $2.01^{\mathrm{b}} \pm 0.10$ & $8.48^{c} \pm 0.68$ \\
\hline $5^{\text {th }}$ week & & $444.6^{b} \pm 1.51$ & $969.9^{a} \pm 1.22$ & $2.25^{\mathrm{a}} \pm 0.07$ & $9.02^{b} \pm 0.37$ \\
\hline p-value & & 0.000 & 0.005 & 0.001 & 0.002 \\
\hline \multicolumn{6}{|c|}{ Probiotics concentrations by contact times interactions } \\
\hline \multirow[t]{5}{*}{$0.5 \mathrm{~g} / \mathrm{L}$} & $1^{\text {st }}$ week & $121.5^{\mathrm{e}} \pm 1.25$ & $140.3^{e} \pm 0.42$ & $1.15^{c} \pm 0.01$ & $1.32^{\mathrm{e}} \pm 0.02$ \\
\hline & $2^{\text {nd }}$ week & $402.3^{c} \pm 4.32$ & $318.6^{d} \pm 0.98$ & $0.79^{d} \pm 0.00$ & $7.01^{c} \pm 0.11$ \\
\hline & $3^{\text {rd }}$ week & $510.5^{a} \pm 1.73$ & $613.0^{\complement} \pm 0.68$ & $1.20^{c} \pm 0.03$ & $8.89^{\mathrm{a}} \pm 0.33$ \\
\hline & $4^{\text {th }}$ week & $315.3^{d} \pm 5.05$ & $800.6^{b} \pm 1.28$ & $2.56^{\mathrm{a}} \pm 0.10$ & $5.44^{d} \pm 0.29$ \\
\hline & $5^{\text {th }}$ week & $473.5^{b} \pm 29.96$ & $1030.8^{\mathrm{a}} \pm 1.35$ & $2.22^{\mathrm{b}} \pm 0.15$ & $8.53^{b} \pm 0.64$ \\
\hline \multirow[t]{5}{*}{$1.0 * \mathrm{~g} / \mathrm{L}$} & $1^{\text {st }}$ week & $107.5^{\mathrm{d}} \pm 1.36$ & $130.8^{\mathrm{e}} \pm 1.01$ & $1.21^{\mathrm{c}} \pm 0.01$ & $1.14^{\mathrm{e}} \pm 0.02$ \\
\hline & $2^{\text {nd }}$ week & $415.0^{\mathrm{b}} \pm 4.69$ & $299.6^{d} \pm 1.54$ & $0.72^{d} \pm 0.01$ & $7.67^{c} \pm 0.15$ \\
\hline & $3^{\text {rd }}$ week & $526.3^{a} \pm 7.61$ & $601.1^{\complement} \pm 0.94$ & $1.14^{\mathrm{c}} \pm 0.01$ & $9.46^{b} \pm 0.19$ \\
\hline & $4^{\text {th }}$ week & $331.1^{c} \pm 1.54$ & $789.5^{b} \pm 3.43$ & $2.39^{a} \pm 0.07$ & $5.92^{\mathrm{d}} \pm 0.21$ \\
\hline & $5^{\text {th }}$ week & $536.0^{\mathrm{a}} \pm 2.06$ & $994.8^{a} \pm 1.62$ & $1.87^{\mathrm{b}} \pm 0.07$ & $10.5^{\mathrm{a}} \pm 0.46$ \\
\hline \multirow[t]{5}{*}{$1.5 \mathrm{~g} / \mathrm{L}$} & $1^{\text {st }}$ week & $103.0^{c} \pm 1.71$ & $117.6^{e} \pm 0.88$ & $1.14^{\mathrm{b}} \pm 0.02$ & $1.18^{\mathrm{e}} \pm 0.03$ \\
\hline & $2^{\text {nd }}$ week & $434.6^{b} \pm 4.05$ & $285.3^{d} \pm 1.43$ & $0.65^{d} \pm 0.00$ & $8.67^{d} \pm 0.13$ \\
\hline & $3^{\text {rd }}$ week & $589.4^{\mathrm{a}} \pm 1.67$ & $576.1^{\complement} \pm 1.40$ & $0.9^{\complement} \pm 0.03$ & $11.9^{\mathrm{a}} \pm 0.52$ \\
\hline & $4^{\text {th }}$ week & $454.2^{b} \pm 2.65$ & $749.6^{\mathrm{b}} \pm 1.02$ & $1.68^{\mathrm{b}} \pm 0.11$ & $9.80^{\mathrm{b}} \pm 0.62$ \\
\hline & $5^{\text {th }}$ week & $423.0^{\mathrm{b}} \pm 3.88$ & $901.3^{a} \pm 0.71$ & $2.21^{\mathrm{a}} \pm 0.19$ & $9.62^{c} \pm 0.99$ \\
\hline \multirow[t]{5}{*}{$2.0 \mathrm{~g} / \mathrm{L}$} & $1^{\text {st }}$ week & $128.3^{d} \pm 2.01$ & $111.6^{\mathrm{e}} \pm 1.20$ & $0.87^{\mathrm{d}} \pm 0.02$ & $1.84^{\mathrm{e}} \pm 0.06$ \\
\hline & $2^{\text {nd }}$ week & $385.0^{c} \pm 4.67$ & $261.1^{d} \pm 2.44$ & $0.67^{\mathrm{e}} \pm 0.01$ & $8.06^{d} \pm 0.21$ \\
\hline & $3^{\text {rd }}$ week & $557.1^{\mathrm{b}} \pm 1.30$ & $540.6^{c} \pm 1.60$ & $0.97^{c} \pm 0.02$ & $11.39^{\mathrm{b}} \pm 0.39$ \\
\hline & $4^{\text {th }}$ week & $629.1^{\mathrm{a}} \pm 1.24$ & $724.1^{b} \pm 1.27$ & $1.15^{\mathrm{b}} \pm 0.02$ & $14.96^{\mathrm{a}} \pm 0.40$ \\
\hline & $5^{\text {th }}$ week & $410.0^{c} \pm 2.12$ & $882.6^{a} \pm 2.78$ & $2.18^{\mathrm{a}} \pm 0.11$ & $9.91^{c} \pm 0.57$ \\
\hline \multirow[t]{5}{*}{ Control } & $1^{\text {st }}$ week & $119.6^{c} \pm 1.08$ & $145.0^{\mathrm{e}} \pm 0.00$ & $1.21^{\mathrm{c}} \pm 0.01$ & $1.25^{\mathrm{e}} \pm 0.02$ \\
\hline & $2^{\text {nd }}$ week & $378.8^{b} \pm 3.64$ & $334.1^{\mathrm{d}} \pm 2.89$ & $0.88^{d} \pm 0.01$ & $6.01^{d} \pm 0.09$ \\
\hline & $3^{\text {rd }}$ week & $505.6^{a} \pm 7.07$ & $621.1^{c} \pm 1.92$ & $1.22^{\mathrm{c}} \pm 0.02$ & $8.46^{\mathrm{a}} \pm 0.16$ \\
\hline & $4^{\text {th }}$ week & $360.3^{b} \pm 1.52$ & $805.1^{b} \pm 1.57$ & $2.25^{\mathrm{b}} \pm 0.09$ & $6.29^{c} \pm 0.30$ \\
\hline & $5^{\text {th }}$ week & $380.8^{b} \pm 1.94$ & $1040.0^{\mathrm{a}} \pm 3.41$ & $2.76^{a} \pm 0.13$ & $6.54^{b} \pm 0.38$ \\
\hline p-value & & 0.000 & 0.001 & 0.000 & 0.001 \\
\hline
\end{tabular}

Means carrying different superscripts in the same column are significantly different at $(p \leq 0.05)$ or highly significantly different at $(p<0.01)$. Means carrying the same superscripts in the same column are non-significantly different at $\mathrm{P}<0.05$. LBW $=$ Live body weight, $\mathrm{BWG}=$ Body weight gain, $\mathrm{FCR}=$ Feed conversion ratio, $\mathrm{PI}=$ Performance index, $\mathrm{SE}=$ Standard error, *Recommended concentration by the manufacturer 
revealed highly significant increases $(\mathrm{p}<0.01)$ in the broilers supplemented with $2.0 \mathrm{~g} / \mathrm{L} B$. subtilis suspension compared to other supplemented groups and the unsupplemented control group, with no significant differences between heart weights of the broilers supplemented with 2.0 and $1.5 \mathrm{~g} / \mathrm{L}$ B. subtilis. On the other hand, spleen's weights revealed highly significant declines $(\mathrm{p}<0.01)$ in all treated broilers compared to the unsupplemented control group.

\section{Intestinal microbial load and immunoglobulin concentration}

The effects of different concentrations of $B$. subtilis suspension on the intestinal microbial load and immunoglobulin concentration of broilers are shown in Table-4. The total bacterial counts revealed highly significant declines $(\mathrm{p}<0.01)$ in broilers supplemented with $2.0 \mathrm{~g} / \mathrm{L} B$. subtilis, with no significant differences between broilers supplemented with 2.0 and $1.5 \mathrm{~g} / \mathrm{L}$ B. subtilis. Meanwhile, the total Enterobacteriaceae and Salmonella counts showed highly significant declines $(\mathrm{p}<0.01)$ in broilers supplemented $2.0 \mathrm{~g} / \mathrm{L}$ $B$. subtilis compared with the other supplemented groups and the control group.

Immunoglobulin IgG, IgM, and IgA concentrations were found to be significantly increased $(\mathrm{p}<0.01)$ in broilers supplemented with $2.0 \mathrm{~g} / \mathrm{L}$ B. subtilis compared to the other supplemented groups and the control group.

\section{Biochemical profile}

The effects of different concentrations of $B$. subtilis suspension on the biochemical profiles of broilers are shown in Table-5. Total protein, albumin, alanine aminotransferase, and aspartate aminotransferase levels were found to be significantly improved $(\mathrm{p}<0.01)$ in broilers supplemented with $2.0 \mathrm{~g} / \mathrm{L} \mathrm{B}$. subtilis compared to the other treated groups and the untreated controls. Meanwhile, urea and creatinine levels showed a significant decline $(\mathrm{p}<0.01)$ in broilers supplemented with $2.0 \mathrm{~g} \mathrm{~B}$. subtilis/L drinking water compared to the other supplemented groups and the control group.

\section{Histopathological examination}

The results of the histopathological examination after supplementation with different concentrations of B. subtilis suspension are shown in Figures-1-5. The photomicrographs of the livers of broilers supplemented with $0.5 \mathrm{~g} / \mathrm{L}$ (Figure-1a) and $1.0^{*} \mathrm{~g} / \mathrm{L}$ (Figure-1b) B. subtilis suspensions reveal adhesive perihepatitis with severe leukocytic infiltrations, and hepatocyte examination revealed severe vacuolation of the cytoplasm with mild areas of hemorrhage compared to control (Figure-1e). Broilers supplemented with $1.5 \mathrm{~g} / \mathrm{L}$ B. subtilis suspension (Figure-1c)

Table-3: Live body, carcass, and immune organs' weight (mean $\pm \mathrm{SE} / \mathrm{g}$ ) in broilers supplemented with different concentrations of probiotics.

\begin{tabular}{|c|c|c|c|c|c|c|c|}
\hline \multirow[t]{2}{*}{ Probiotic g/L } & \multirow[t]{2}{*}{ LBW $/ g$} & \multirow[t]{2}{*}{ Carcass weight/g } & \multicolumn{2}{|c|}{$\begin{array}{l}\text { Edible organs } \\
\text { weights/g }\end{array}$} & \multicolumn{3}{|c|}{ Immune organs weights/g } \\
\hline & & & Liver/g & Heart/g & Bursa/g & Spleen/g & Thymus/g \\
\hline \multicolumn{8}{|c|}{ Overall means concerning probiotic concentrations } \\
\hline $0.5 \mathrm{~g} / \mathrm{L}$ & $1876^{d} \pm 4.63$ & $1555^{\mathrm{d}} \pm 4.6$ & $20.5^{\mathrm{d}} \pm 0.18$ & 10.3 & $1.45^{\mathrm{e}} \pm 0.01$ & $1.51^{\mathrm{e}} \pm 0.04$ & $2.51^{b, c} \pm 0.08$ \\
\hline $1.0 * \mathrm{~g} / \mathrm{L}$ & $1923^{c} \pm 6.85$ & $1692^{c} \pm 6.8$ & $21.7^{c} \pm 0.22$ & $11.5^{c} \pm 0.09$ & $1.78^{\mathrm{d}} \pm 0.01$ & $1.74^{c} \pm 0.06$ & $2.10^{\mathrm{d}} \pm 0.03$ \\
\hline $1.5 \mathrm{~g} / \mathrm{L}$ & $2102^{b} \pm 14.39$ & $1892^{\mathrm{b}} \pm 14.3$ & $23.1^{\mathrm{b}} \pm 0.16$ & $12.9^{\mathrm{b}} \pm 0.05$ & $1.86^{c} \pm 0.04$ & $1.61^{\mathrm{d}} \pm 0.01$ & $2.45^{c} \pm 0.02$ \\
\hline $2.0 \mathrm{~g} / \mathrm{L}$ & $2191^{\mathrm{a}} \pm 12.00$ & $1996^{\mathrm{a}} \pm 12.0$ & $24.8^{\mathrm{a}} \pm 0.16$ & $14.5^{\mathrm{a}} \pm 0.03$ & $2.33^{a} \pm 0.05$ & $2.46^{b} \pm 0.07$ & $2.97^{\mathrm{a}} \pm 0.06$ \\
\hline Control & $1789^{\mathrm{e}} \pm 6.21$ & $1433^{e} \pm 6.2$ & $19.2^{\mathrm{e}} \pm 0.09$ & $9.2^{\mathrm{e}} \pm 0.02$ & $2.12^{\mathrm{b}} \pm 0.05$ & $2.62^{\mathrm{a}} \pm 0.01$ & $2.56^{b} \pm 0.03$ \\
\hline$p$-value & 0.000 & 0.000 & 0.000 & 0.003 & 0.001 & 0.001 & 0.001 \\
\hline
\end{tabular}

Means carrying different superscripts in the same column are significantly different at $P \leq 0.05$ or highly significantly different at $P<0.01$. Means carrying the same superscripts in the same column are non-significantly different at $P>0.05$. LBW=Live body weight, SE=Standard error, *Recommended concentration by the manufacturer

Table-4: Logarithmic bacterial counts (mean $\pm \mathrm{SE} \mathrm{CFU} / \mathrm{mL}$ ) and Immunoglobulin concentrations (mean $\pm \mathrm{SE} \mathrm{mg} / \mathrm{dL}$ ) in broilers supplemented with different concentrations of probiotics.

\begin{tabular}{|c|c|c|c|c|c|c|}
\hline \multirow[t]{2}{*}{ Probiotic g/L } & \multicolumn{3}{|c|}{ Log. bacterial counts } & \multicolumn{3}{|c|}{ Immunoglobulin concentrations } \\
\hline & $\begin{array}{l}\text { Log TBC } \\
\text { CFU/mL }\end{array}$ & $\begin{array}{l}\text { Log TEC } \\
\text { CFU/mL }\end{array}$ & $\begin{array}{l}\text { Log TSC } \\
\text { CFU/mL }\end{array}$ & $\begin{array}{c}\text { IgG } \\
\mathrm{mg} / \mathrm{dL}\end{array}$ & $\underset{\mathrm{Ig} / \mathrm{dL}}{\mathrm{IgM}}$ & $\begin{array}{c}\text { IgA } \\
\mathrm{mg} / \mathrm{dL}\end{array}$ \\
\hline \multicolumn{7}{|c|}{ Overall means concerning probiotic concentrations } \\
\hline $0.5 \mathrm{~g} / \mathrm{L}$ & $4.20^{\mathrm{b}} \pm 0.01$ & $3.50^{\mathrm{a}} \pm 0.05$ & $2.40^{\mathrm{a}} \pm 0.01$ & $1359^{d} \pm 0.01$ & $254^{\mathrm{d}} \pm 0.00$ & $143^{c} \pm 0.01$ \\
\hline $1.0 * \mathrm{~g} / \mathrm{L}$ & $4.14^{c} \pm 0.00$ & $3.45^{\mathrm{b}} \pm 0.01$ & $2.32^{\mathrm{b}} \pm 0.00$ & $1495^{c} \pm 0.02$ & $300^{c} \pm 0.00$ & $158^{\mathrm{b}} \pm 0.01$ \\
\hline $1.5 \mathrm{~g} / \mathrm{L}$ & $4.06^{d} \pm 0.02$ & $3.42^{c} \pm 0.03$ & $1.43^{c} \pm 0.00$ & $1540^{\mathrm{b}} \pm 0.02$ & $406^{b} \pm 0.01$ & $155^{\mathrm{b}} \pm 0.01$ \\
\hline $2.0 \mathrm{~g} / \mathrm{L}$ & $4.06^{d} \pm 0.02$ & $2.70^{d} \pm 0.04$ & $0.60^{\mathrm{d}} \pm 0.01$ & $1794^{\mathrm{a}} \pm 0.01$ & $499^{a} \pm 0.00$ & $243^{a} \pm 0.02$ \\
\hline Control & $4.74^{a} \pm 0.01$ & $2.49^{e} \pm 0.03$ & $0.47^{e} \pm 0.01$ & $1318^{d} \pm 0.02$ & $204^{\mathrm{e}} \pm 0.01$ & $153^{\mathrm{b}} \pm 0.01$ \\
\hline p-value & 0.001 & 0.001 & 0.001 & 0.000 & 0.000 & 0.001 \\
\hline
\end{tabular}

Means carrying different superscripts in the same column are significantly different at $P \leq 0.05$ or highly significantly different at $P<0.01$. Means carrying the same superscripts in the same column are non-significantly different at $P<0.05$. $\mathrm{TBC}=$ Total bacterial count, TEC=Total Enterobacteriaceae count, TSC=Total Salmonella count, IgG=Immunoglobulin G, IgM=Immunoglobulin M, IgA=Immunoglobulin A, Log=Logarithm, SE=Standard error, *Recommended concentration by the manufacturer 
Table-5: Biochemical parameters (mean \pm SE) in broilers supplemented with different concentrations of probiotics.

\begin{tabular}{lcccccc}
\hline Probiotic g/L & TP g/dL & ALB g/dL & ALT IU/L & AST IU/L & Urea $\mathbf{~ g / d L ~}$ & Creat $\mathbf{~ m g / d L ~}$ \\
\hline Overall means concerning probiotic concentrations & & & & \\
$0.5 \mathrm{~g} / \mathrm{L}$ & $3.5^{\mathrm{d}} \pm 0.00$ & $1.87^{\mathrm{d}} \pm 0.00$ & $27.1^{\mathrm{b}} \pm 0.02$ & $36.1^{\mathrm{b}} \pm 0.02$ & $26.4^{\mathrm{b}} \pm 0.23$ & $0.56^{\mathrm{c}} \pm 0.01$ \\
$1.0^{*} \mathrm{~g} / \mathrm{L}$ & $4.39^{\mathrm{b}} \pm 0.02$ & $3.17^{\mathrm{b}} \pm 0.00$ & $26.9^{\mathrm{c}} \pm 0.05$ & $36.1^{\mathrm{b}} \pm 0.09$ & $25.2^{\mathrm{c}} \pm 0.13$ & $0.74^{\mathrm{a}} \pm 0.01$ \\
$1.5 \mathrm{~g} / \mathrm{L}$ & $4.9^{\mathrm{b}} \pm 0.01$ & $2.37^{\mathrm{c}} \pm 0.01$ & $25.7^{\mathrm{d}} \pm 0.08$ & $34.8^{\mathrm{c}} \pm 0.13$ & $25.0^{\mathrm{c}} \pm 0.05$ & $0.49^{\mathrm{d}} \pm 0.02$ \\
$2.0 \mathrm{~g} / \mathrm{L}$ & $4.99^{\mathrm{a}} \pm 0.01$ & $3.87^{\mathrm{a}} \pm 0.01$ & $27.4^{\mathrm{a}} \pm 0.23$ & $36.6^{\mathrm{a}} \pm 0.07$ & $24.3^{\mathrm{d}} \pm 0.33$ & $0.44^{\mathrm{e}} \pm 0.01$ \\
Control & $3.5^{\mathrm{c}} \pm 0.01$ & $1.87^{\mathrm{d}} \pm 0.00$ & $27.2^{\mathrm{b}} \pm 0.08$ & $36.1^{\mathrm{b}} \pm 0.10$ & $27.2^{\mathrm{a}} \pm 0.13$ & $0.59^{\mathrm{b}} \pm 0.01$ \\
p-value & 0.005 & 0.001 & 0.000 & 0.006 & 0.000 & 0.000 \\
\hline
\end{tabular}

Means carrying different superscripts in the same column are significantly different at $\mathrm{P} \leq 0.05$ or highly significantly different at $P<0.01$. Means carrying the same superscripts in the same column are non-significantly different at $\mathrm{P}>0.05$. $\mathrm{TP}=$ Total protein, $\mathrm{ALB}=\mathrm{Albumin}, \mathrm{ALT}=\mathrm{Alanine}$ aminotransferase, $\mathrm{AST}=\mathrm{Aspartate}$ aminotransferase, Urea $=\mathrm{Urea}$, Creat $=$ Creatinine, $\mathrm{SE}=$ Standard error, $*$ Recommended concentration by the manufacturer

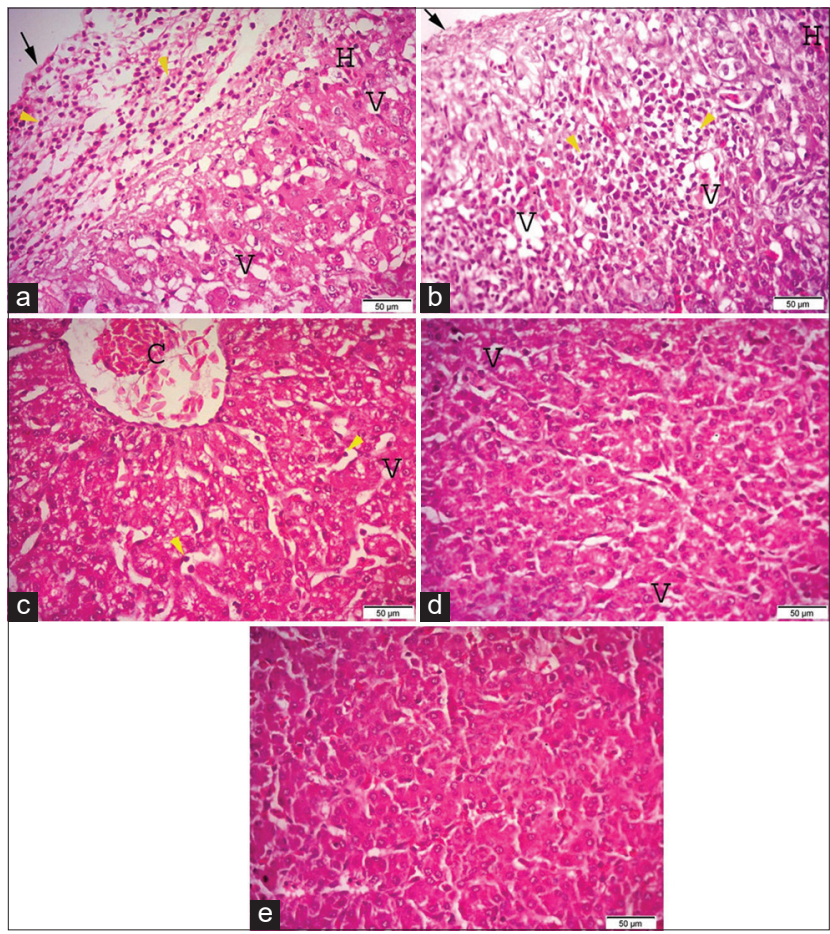

Figure-1: Photomicrographs of hematoxylin and eosin (H\&E) stained sections of the liver, (a) the liver of G1 (broilers supplemented with $0.5 \mathrm{~g} / \mathrm{L}$ Bacillus subtilis) showing adhesive perihepatitis (arrow), mononuclear cell infiltration (arrowhead), vaculation of hepatocytes cytoplasm (V) with mild hemorrhage $(H)$. (b) The liver of G2 (broilers supplemented with $1.0^{*} \mathrm{~g} / \mathrm{L}$ B. subtilis). (c) The liver of G3 (broilers supplemented with $1.5 \mathrm{~g} / \mathrm{L}$ B. subtilis). (d) The liver of G4 (2.0 g/L B. subtilis). (e) The liver of G5 (control group). H\&E (40x). Bar $50 \mu \mathrm{m}$.

showed vacuolated cytoplasm in the liver, congestion of the central vein, and mild leukocytic infiltrations compared to the controls (Figure-1e). Meanwhile, the livers of broilers supplemented with $2.0 \mathrm{~g} / \mathrm{L} \mathrm{B}$. subtilis (Figure-1d) showed pronounced improvement of the histopathological picture.

The histopathological examinations of the heart (Figures-2a and $\mathrm{b}$ ) revealed severe fibrinous pericarditis with degeneration of the myocardium, and some of the myocardial cells showed vacuolated cytoplasm with leukocytic infiltrations in broilers supplemented with 0.5 and $1.0^{*} \mathrm{~g} / \mathrm{L}$ B. subtilis, respectively, compared to the controls (Figure-2e). The hearts of the broilers supplemented with $1.5 \mathrm{~g} / \mathrm{L}$

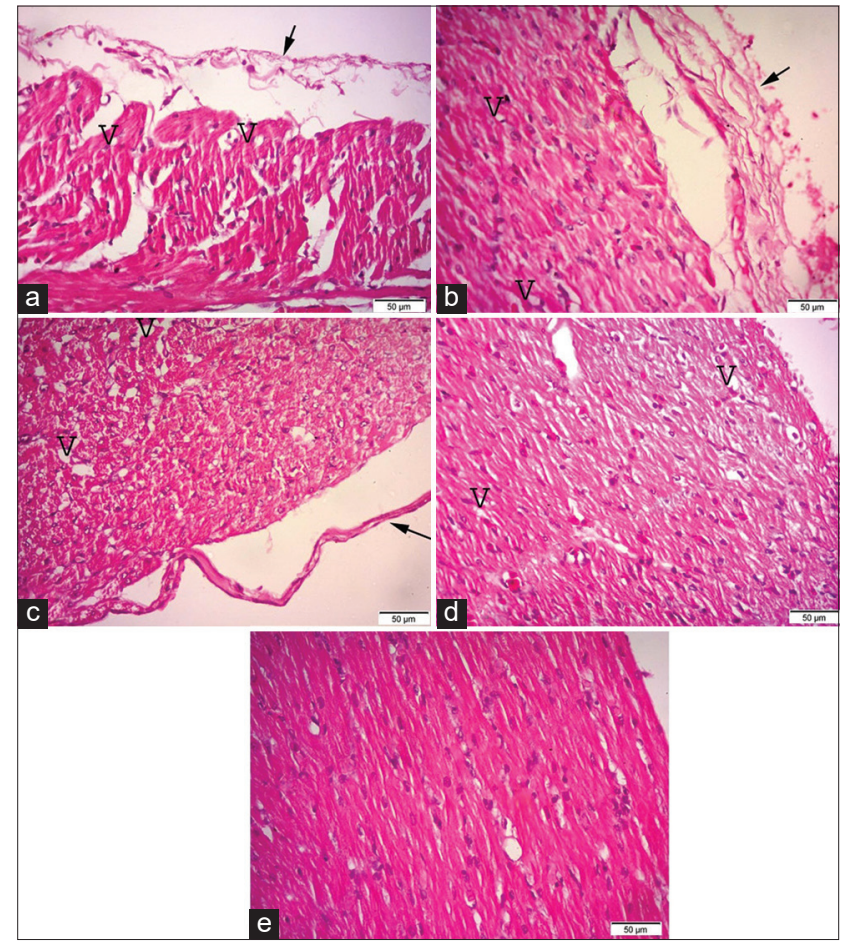

Figure-2: Photomicrographs of hematoxylin and eosin (H\&E) stained sections of the heart, (a) the heart of G1 (broilers supplemented with $0.5 \mathrm{~g} / \mathrm{L}$ Bacillus subtilis) showing pericarditis (arrow), vaculation of myocardial cells (V). (b) The heart of G2 (broilers supplemented with 1.0* $\mathrm{g} / \mathrm{L}$ B. subtilis). (c) The heart of G3 (broilers supplemented with $1.5 \mathrm{~g} / \mathrm{L} B$. subtilis). (d) The heart of G4 (broilers supplemented with $2.0 \mathrm{~g} / \mathrm{L} \mathrm{B}$. subtilis). (e) The heart of G5 (control group). H\&E (40x). Bar $50 \mu \mathrm{m}$.

B. subtilis (Figure-2c) showed mild pericarditis with mild myocarditis and vacuolated cytoplasm in some of the myocardial cells. The hearts of the broilers supplemented with $2.0 \mathrm{~g} / \mathrm{L}$ B. subtilis (Figure-2d) showed an improved and near-normal histopathological picture.

Stained histopathological sections of the bursas of Fabricius (Figures-3a and b), thymuses (Figures-4a and $b$ ), and spleens (Figures-5a and b) of the broilers supplemented with 0.5 and $1.0^{*} \mathrm{~g} / \mathrm{L}$ B. subtilis, respectively, revealed severe lymphoid depletion compared to the controls (bursa of Fabricius: Figure-3e, thymus: Figure-4e, spleen: Figure-5e). Broilers supplemented with $1.5 \mathrm{~g} / \mathrm{L}$ B. subtilis showed mild lymphoid depletion in the bursa of Fabricius (Figure-3c), thymus 


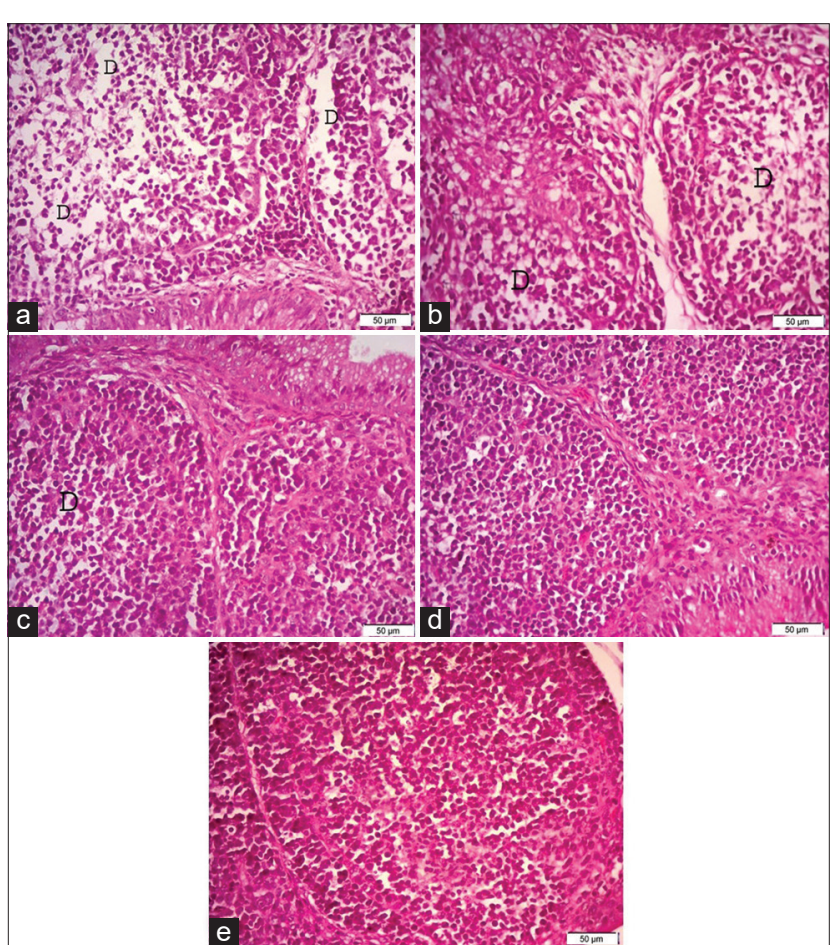

Figure-3: Photomicrographs of hematoxylin and eosin (H\&E) stained sections of the bursa of Fabricius, (a) the bursa of Fabricius of G1 (broiler supplemented with $0.5 \mathrm{~g} / \mathrm{L}$ Bacillus subtilis) showing severe depletion of lymphoid follicles (D). (b) The bursa of Fabricius of G2 (broiler supplemented with $1.0 * \mathrm{~g} / \mathrm{L} B$. subtilis). (c) The bursa of Fabricius of G3 (broiler supplemented with 1.5 $\mathrm{g} / \mathrm{L}$ B. subtilis). (d) The bursa of Fabricius of G4 (broiler supplemented with $2.0 \mathrm{~g} / \mathrm{L} B$. subtilis). (e) The bursa of G5 (control group). H\&E. $(40 \times)$. Bar $50 \mu \mathrm{m}$.

(Figure-4c), and spleen (Figure-5c). The bursas of Fabricius, thymuses, and spleens of the broilers that were supplemented with $2.0 \mathrm{~g} / \mathrm{L} \mathrm{B}$. subtilis showed lymphoid hyperplasia (Figures-3d-5d) compared to the normal view of the bursa of Fabricius in the controls (Figure-3e, thymus in Figure-4e, and spleen in Figure-5e).

\section{Discussion}

Probiotics are effective and promising feed and water additives in the field of preventive measures and therapeutics for broiler chickens [31,32]. Probiotics can enhance and improve the intestinal mucosa and microbiota, thus improving the performance and production of broiler chickens $[33,34]$. B. subtilis has been categorized as a type of probiotic bacteria that naturally inhabit the intestine of healthy broilers and can promote gut conditions [35,36], absorption functions, performance [37,38], and immunity [39], and can alleviate many overwhelming challenges, such as microbial stress and heat stress [40].

The current in vitro results revealed that the B. subtilis $2.0 \mathrm{~g} / \mathrm{L}$ suspension was able to produce direct significant in vitro antimicrobial action against $E$. coli O157: $\mathrm{H} 7\left(4.5 \times 10^{12} \mathrm{CFU} / \mathrm{mL}\right)$ and $S$. Typhimurium $\left(1.2 \times 10^{7} \mathrm{CFU} / \mathrm{mL}\right)$ after 0.25 and $2 \mathrm{~h}$, respectively. Supplementing broilers with 1.5 or $2.0 \mathrm{~g} \mathrm{~B}$. subtilis

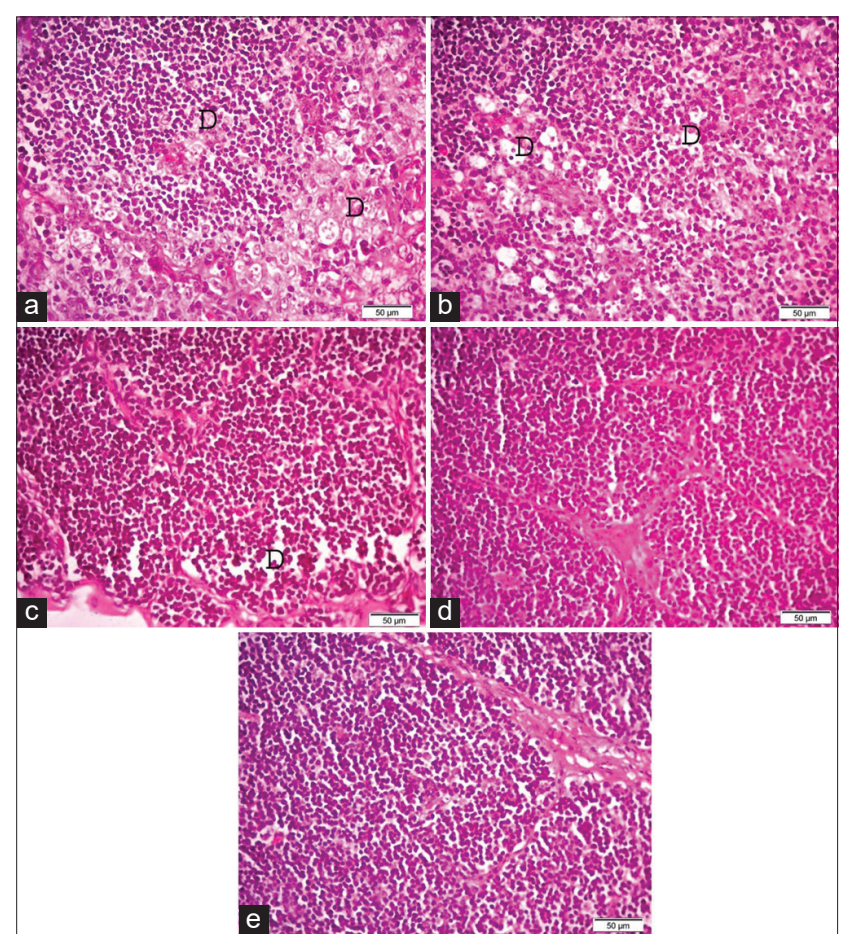

Figure-4: Photomicrographs of hematoxylin and eosin (H\&E) stained sections of the thymus (a) the thymus of G1 (broilers supplemented with $0.5 \mathrm{~g} / \mathrm{L}$ Bacillus subtilis) showing severe depletion of lymphoid follicles (D). (b) The thymus of G2 (broilers supplemented with $1.0 * \mathrm{~g} / \mathrm{L}$ B. subtilis). (c) The thymus of G3 (broilers supplemented with $1.5 \mathrm{~g} / \mathrm{L}$ B. subtilis). (d) The thymus of G4 (broilers supplemented with $2.0 \mathrm{~g} / \mathrm{L} \mathrm{B}$. subtilis). (e) The thymus of G5 (control group). H\&E. (40×). Bar $50 \mu \mathrm{m}$.

$\left(5 \times 10^{6} \mathrm{CFU} / \mathrm{g} / \mathrm{L}\right.$ of drinking water) induced significant reductions in total bacterial, Enterobacteriaceae, and Salmonella counts. These in vitro and in vivo antimicrobial actions may be attributable to direct-fed microbes, organic acid production, and protein killing molecules released from $B$. subtilis. The current results were consistent with those in the study by $\mathrm{Oh}$ et al. [41], who found that supplementing broilers with probiotics reduced coliform and E. coli counts in Salmonella-challenged broilers. Ebrahimi et al. [42] concluded that acidic $\mathrm{pH}$ from organic acids produced by supplementing broilers with PrimaLac ${ }^{\circledR}(120 \mathrm{~g} / 1$ $\mathrm{L}$ water until the $14^{\text {th }}$ day, $454 \mathrm{~g} / 1000 \mathrm{~kg}$ ration until the $28^{\text {th }}$ day, and $225 \mathrm{~g} / 1000 \mathrm{~kg}$ ration for the rest of the growing period) reduced growth and colonization of Campylobacter jejuni. Nishiyama et al. [43] and Saint-Cyr et al. [44] reported that using Lactobacillus gasseri SBT2055 and Bacillus sp. suppressed the growth and colonization of $C$. jejuni. Carter et al. [45] reported similar results when they used a mixture of Enterococcus faecium and Lactobacillus salivarius to inhibit the growth and colonization of $S$. Enteritidis. Neveling et al. [46] revealed that multispecies probiotics composed of Lactobacillus crispatus, L. salivarius, Lactobacillus gallinarum, Lactobacillus johnsonii, Enterococcus faecalis, and Bacillus amyloliquefaciens could inhibit the colonization of $S$. Enteritidis in the intestine when administered to broiler chickens. 


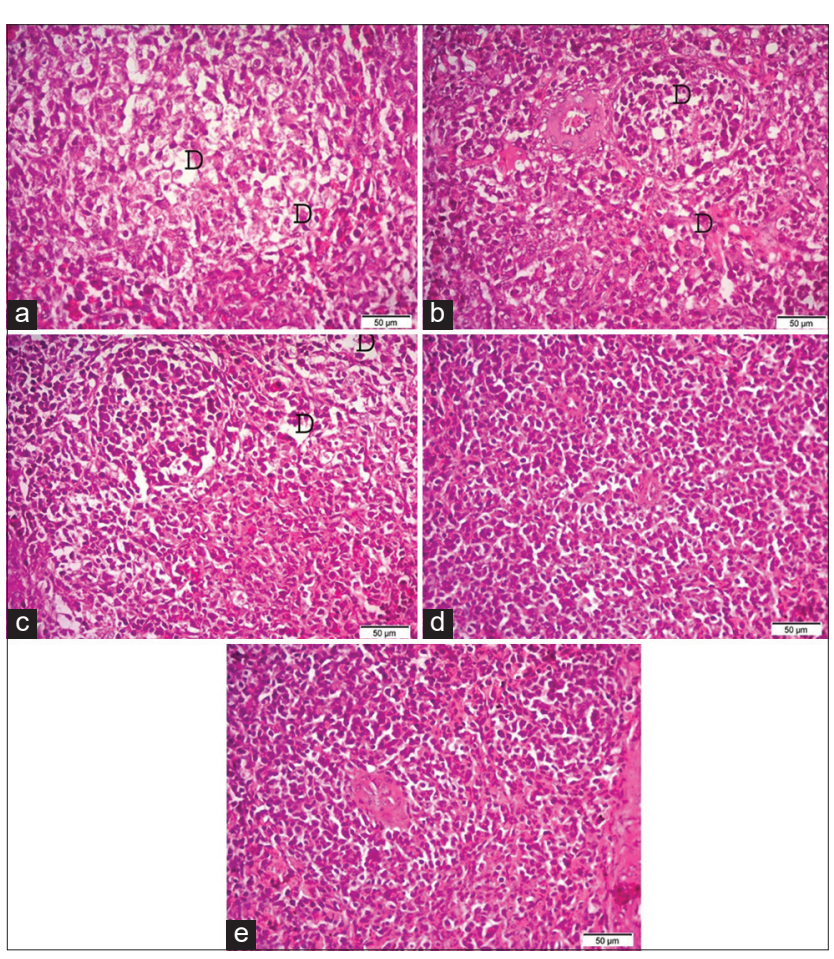

Figure-5: Photomicrographs of hematoxylin and eosin (H\&E) stained sections of the spleen (a) the spleen of G1 (broilers supplemented with $0.5 \mathrm{~g} / \mathrm{L}$ Bacillus subtilis) showing severe depletion of lymphoid follicles (D). (b) The spleen of G2 (broilers supplemented with $1.0 * \mathrm{~g} / \mathrm{L}$ $B$. subtilis). (c) The spleen of $\mathrm{G} 3$ (broilers supplemented with $1.5 \mathrm{~g} / \mathrm{L}$ B. subtilis). (d) The spleen of G4 (broilers supplemented with $2.0 \mathrm{~g} / \mathrm{L} B$. subtilis). (e) The spleen of G5 (control group). H\&E (40×). Bar $50 \mu \mathrm{m}$.

The current results showed that the B. subtilis $2.0 \mathrm{~g} / \mathrm{L}$ suspension was able to produce significant in vitro antimicrobial action against $C$. albicans $\left(2.5 \times 10^{6} \mathrm{CFU} / \mathrm{mL}\right)$ and $T$. mentagrophytes $\left(2.5 \times 10^{6}\right.$ $\mathrm{CFU} / \mathrm{mL}$ ) after $2 \mathrm{~h}$. The current results correlate well with those of Zhang et al. [47], who reported that B. subtilis ANSB060 could neutralize the aflatoxins produced by Aspergillus pseudotamarii, Aspergillus flavus, Aspergillus niger, Aspergillus parasiticus, Aspergillus ochraceoroseus, and Aspergillus nomius and, when administered to broilers, could improve growth and increase weight gains. Fan et al. [48] found that the inclusion of $B$. subtilis in the diets of broilers could improve the intestinal microbiota and neutralize the harmful influence of aflatoxin-producing agents by degrading the cell wall polysaccharide contents of aflatoxin-producing agents. Abdolmaleki et al. [49] reported that probiotic Bacillus spp. MBIA2.40 had a protective influence. They found that the inclusion of Bacillus spp. MBIA2.40 in diets contaminated with fungal growth and their associated aflatoxins could minimize the negative influences of these fungal organisms and their toxins in broiler chickens.

Probiotics can exert their action through various methods, including blocking bacterial binding sites (competitive inhibition), regenerating intestinal mucosa, and enhancing the secretion of digestive enzymes. In our study, supplementing broilers with
1.5 and $2.0 \mathrm{~g} \mathrm{~B}$. subtilis $\left(5 \times 10^{6} \mathrm{CFU} / \mathrm{g}\right.$ in each liter of drinking water) enhanced the live body weight, accelerated weight gains, and improved performance indices compared to $1.0^{*} \mathrm{~g}$ B. subtilis (the quantity recommended by the manufacturer). The observed actions could be attributed to the enhancing influence of $B$. subtilis probiotics on intestinal permeability and absorption functions. The results were consistent with those of He et al. [50] and Hosseini et al. [51], who reported that the inclusion of probiotics in broiler chicken rations could improve nutrient digestion, performance, antioxidant levels and activity, and intestinal morphology barriers against pathogenic microorganisms. Sobczak and Kozłowski [52] recorded synchronized results and found that $B$. subtilis $\left(1 \times 10^{8} \mathrm{CFU} / \mathrm{kg}\right.$ feed) improved performance, egg quality, and yolk cholesterol contents. Kim and Lillehoi [53] concluded in their study that probiotics can be beneficial for the growth and performance of broiler chickens and could enhance disease resistance. Ribeiro et al. [54], Buta et al. [55], Meyer et al. [56], and Dong et al. [57] found that B. subtilis supplementation could alter the intestinal permeability and enhance the productive performances of broiler chickens.

In our study, the broilers that were supplemented with 1.5 and $2.0 \mathrm{~g}$ B. subtilis $\left(5 \times 10^{6} \mathrm{CFU} / \mathrm{g}\right.$ in each liter of drinking water) showed increased carcass weights and increased edible and immune organ weights. The results were supported by those of Hrnčár et al. [58], who used a 20-g Bacillus amyloliquefaciens $/ \mathrm{kg}$ ration for 35 days and found enhanced performance, carcass quality, and improved digestibility. Hassan et al. [59] concluded that using Bacillus sp., Clostridium butyricum probiotics $(0.05 \%$ Saltose, $0.05 \%$ Clostat, and $0.05 \%$ Clostridium-stop), and phytobiotics (0.1\% Sangrovit) significantly improved carcass traits compared to antibiotics $(0.025 \%$ bacitracin methylene di-salicylate). Javandel et al. [60] investigated the influence of powdered Heracleum persicum and probiotic combinations on the performance and carcass quality of 270 1-day-old broilers and revealed significantly higher body weight gains, body weights, and carcass weights, with significantly lower abdominal fat.

Probiotics can produce immune-stimulant activity by impacting $\mathrm{T}$ and $\mathrm{B}$ effector cells, $\mathrm{T}$ cell regulators, enterocytes, and antigen-presenting cells, as reported by Alagawany et al. [61]. The current study revealed an immune-stimulant influence of probiotics in all tested concentrations, but the most prominent increase was recorded in the $\mathrm{IgG}, \mathrm{IgM}$, and IgA concentrations of the broilers supplemented with $2.0 \mathrm{~g} \mathrm{~B}$. subtilis $\left(5 \times 10^{6} \mathrm{CFU} / \mathrm{g} / \mathrm{L}\right.$ of drinking water $)$. The results were consistent with those reported by Harimurti and Ariyadi [62], who found a great ability of probiotics to stimulate Peyer's patches activities, plasma cell functions, and immunoglobulin secretions. They also reported increased expression of claudin-1, -3 , and -5 mRNA in broilers supplemented with probiotics. 
Trani et al. [63] reported the abilities of probiotics to enhance gut mucosal immunity by increasing the levels of secretory IgA. Yisa et al. [64] and Awais et al. [65] concluded that the inclusion of $1 \mathrm{~g}$ of probiotics in the diet can be sufficient for stimulating the immune system and allowed the proliferation of beneficial microorganisms in the gut. Ashraf and Shah [66] reported that probiotics could enhance gut mucosal immunity by increasing the levels of $\operatorname{IgA}$. Gonmei et al. [67] concluded that Lactobacillus reuteri PIA16, previously isolated from the chicken gut, could enhance the humoral and cell-mediated immunity of broiler chickens, and lower the mortality and susceptibility to disease. Sarwar et al. [68] showed that probiotics, when administered with vaccines, at a rate of $2.0 \mathrm{~g} / 500 \mathrm{~mL}$ of water, could induce an improvement in antibody titer.

Broilers supplemented with 1.5-2.0 g B. subtilis $\left(5 \times 10^{6} \mathrm{CFU} / \mathrm{g} / \mathrm{L}\right.$ of drinking water $)$ in our study showed significant increases in total protein, albumin, alanine aminotransferase, and aspartate aminotransferase and significant declines in urea and creatinine. The enhancing influence of probiotic $B$. subtilis on the biochemical profile could be attributable to the regulation of gastrointestinal permeability and enhanced physiological function. The results were compatible with those of Shankar et al. [69], who recorded improved serum levels of total protein, albumin, and high-density lipoproteins and significantly lower serum levels of total cholesterol and low-density lipoproteins in broilers supplemented with $0.1,0.15$, and $0.2 \%$ Saccharomyces cerevisiae. Deraz [70] recorded improved levels of total protein and glucose in broilers supplemented with Lactococcus lactis at a rate of $10^{9} \mathrm{CFU} / \mathrm{mL}$ and Lactobacillus plantarum at a rate of $10^{12} \mathrm{CFU} / \mathrm{mL}$. The current results were consistent with those of Hussein and Selim [71], who investigated the efficiency of $0.5 \%$ dried yeast (S. cerevisiae), $0.5 \%$ multi-strain probiotics (Lactobacillus acidophilus, B. subtilis, and Aspergillus oryzae), and $0.25 \%$ dried yeast and multi-strain probiotics in broilers. They found higher total protein, globulin, and glucose levels in all of the treatments compared to the controls. Hussein et al. [72] recorded significantly lowered levels of alanine aminotransferase and glucose in broilers that were supplemented with $B$. subtilis probiotics.

The histopathological photomicrographs revealed that supplementing broilers with $2.0 \mathrm{~g} / \mathrm{L} \mathrm{B}$. subtilis probiotics could produce improved and near-normal histopathological pictures of livers and hearts with lymphoid hyperplasia in the bursas of Fabricius, thymuses, and spleens of broilers challenged with $E$. coli $\mathrm{O} 157$ : $\mathrm{H} 7$ and $S$. Typhimurium. The current results were consistent with those reported by Abramowicz et al. [73] and Adhikari et al. [74], who revealed that B. subtilis supplementation in broiler chickens was able to enhance the histopathological structure of the intestine, improved intestinal microbiota actions, and enhanced its barriers to reduce the incidence of necrosis. Huang et al. [75] also revealed that supplementing broiler chickens with $C$. butyricum probiotics improved the structure of the intestinal walls, reduced the incidence of necrotic enteritis, and enhanced the local immune response against pathogenic microorganisms. Olnood et al. [76] found that feeding broiler chickens on B. subtilis induced significant increases in total villus area and villus length and minimized the incidence of pathogenic lesions that arose from the challenge with $C$. perfringens. They also recorded near-normal liver histopathological architecture with mild lymphocytic infiltrations among hepatocytes, while in the intestine they recorded normal intestinal villi with mild metaplasia of the columnar epithelium lining the villi into goblet cells. Kogut [77] recommended probiotics in broiler chickens for their modulatory actions on the intestinal microbiota and their ability to enhance the histopathological picture of the intestine.

\section{Conclusion}

B. subtilis probiotic supplementation at a concentration of $5 \times 10^{6} \mathrm{CFU} / \mathrm{g}$ and a rate of $1.5-2.0 \mathrm{~g} / \mathrm{L}$ of drinking water produced a suitable intrinsic environment for enhancing and flourishing the commensal intestinal microbiota, improved the absorption activity, increased weight gains, enhanced the performance, increased the carcass weights, and improved the biochemical parameters in broiler chickens. The supplementation also initiated immune-stimulating action by increasing immunoglobulin concentrations (IgG, IgM, and IgA). B. subtilis probiotics produced significant in vitro antimicrobial action against $E$. coli $\mathrm{O} 157$ : H7 $\left(4.5 \times 10^{12} \mathrm{CFU} / \mathrm{mL}\right), S$. Typhimurium $\left(1.2 \times 10^{7}\right.$ $\mathrm{CFU} / \mathrm{mL}), C$. albicans $\left(2.5 \times 10^{6} \mathrm{CFU} / \mathrm{mL}\right)$, and T. mentagrophytes $\left(2.5 \times 10^{6} \mathrm{CFU} / \mathrm{mL}\right)$, and minimized and/or prevented the colonization of $E$. coli $\mathrm{O} 157: \mathrm{H} 7$ and $S$. Typhimurium in 14-day-old broiler chickens overwhelmed with $E$. coli $\mathrm{O} 157$ : $\mathrm{H} 7\left(4.5 \times 10^{12}\right)$ and $S$. Typhimurium $\left(1.2 \times 10^{7} \mathrm{CFU} / \mathrm{mL}\right)$.

\section{Authors' Contributions}

ESS designed the in vitro and in vivo experimental design, participated, and supervised the execution of the experiment, and contributed to the writing of the manuscript. RTH conducted the histopathological examinations and contributed to the writing of the manuscript. MSA participated in the execution of the experiment and contributed to the writing of the manuscript. All authors have read and approved the final manuscript.

\section{Acknowledgments}

We would like to express our grateful thanking to Prof. MAA Sobieh for his generous directions while conducting the studies, as well to the Community Services and Environmental Development Sector staff, Faculty of Veterinary Medicine, Suez Canal University, Ismailia, Egypt, for their help. The authors did not receive any funds for this study. 


\section{Competing Interests} interests.

The authors declare that they have no competing

\section{Publisher's Note}

Veterinary World remains neutral with regard to jurisdictional claims in published institutional affiliation.

\section{References}

1. Yadav, A.S., Kolluri, G., Gopi, M., Karthik, K., Malik, Y.S. and Dhama, K. (2016) Exploring alternatives to antibiotics as health promoting agents in poultry a review. J. Exp. Biol. Agric. Sci., 4(3S): 368-383.

2. Tiwari, R., Chakraborty, S., Dhama, K., Wani, M.Y., Kumar, A. and Kapoor, S. (2014) Wonder world of phages: Potential biocontrol agents safeguarding biosphere and health of animals and humans current scenario and perspectives. Pak. J. Biol. Sci., 17(3): 316-328.

3. Soliman, E.S., Hamad, R.T. and Ahmed, A. (2017) Prophylactic and immune modulatory influences of Nigella sativa Linn. in broilers exposed to biological challenge. Vet. World, 10(12): 1447-1455.

4. Abd El-Hack, M.E., Alagawany, M., AbdelMoneim, A.M.E., Mohammed, N.G., Khafaga, A.F., BinJumah, M., Othman, S.I., Allam, A.A. and Elnesr, S.S. (2020) Cinnamon (Cinnamomum zeylanicum) oil as a potential alternative to antibiotics in poultry a review. Antibiotics (Basel), 9(5): 210.

5. Soliman, E.S., Mahmoud, F.F., Fadel, M.A. and Hamad, R.T. (2020) Prophylactic impact of nano-selenium on performance, carcasses quality and tissues' selenium concentration using RP-HPLC during microbial challenge in broiler chickens. Vet. World, 13(9): 1780-1797.

6. Aalaei, M., Khatibjoo, A., Zaghari, M., Taherpou, K., Akbari-Gharaei, M. and Soltani, M. (2019) Effect of singleand multi-strain probiotics on broiler breeder performance, immunity and intestinal toll-like receptors expression. $J$. Appl. Anim. Res., 47(1): 236-242.

7. Hill, C., Guarner, F., Reid, G., Gibson, G.R., Merenstein, D.J., Pot, B., Morelli, L., Canani, R.B., Flint, H.J., Salminen, S., Calder, P.C. and Sanders, M.E. (2014) Expert consensus document. The international scientific association for probiotics and prebiotics consensus statement on the scope and appropriate use of the term probiotic. Nat. Rev. Gastroenterol. Hepatol., 11(8): 506-514.

8. Abd El-Hack, M.E., Mahgoub, S.A., Alagawany, M. and Ashour, E.A. (2017) Improving productive performance and mitigating harmful emissions from laying hen excreta via feeding on graded levels of corn DDGS with or without Bacillus subtilis probiotic. J. Anim. Physiol. Anim. Nutr., 101(5): 904-913.

9. Mokhtari, R., Yazdani, A. and Kashfi, H. (2015) The effects of different growth promoters on performance and carcass characteristics of broiler chickens. J. Vet. Med. Anim. Health, 7(8): 271-277.

10. Popova, T. (2017) Effect of probiotics in poultry for improving meat quality. Curr. Opin. Food Sci., 14(1): 72-77.

11. Alagawany, M., Abd El-Hack, M.E., Arif, M. and Ashour, E.A. (2016) Individual and combined effects of crude protein, methionine, and probiotic levels on laying hen productive performance and nitrogen pollution in the manure. Environ. Sci. Pollut. Res., 23(22): 22906-22913.

12. Iannitti, T. and Palmieri, B. (2010) Therapeutical use of probiotic formulations in clinical practice. Clin. Nutr., 29(6): 701-725.

13. Soliman, E.S., Sallam, N.H. and Abouelhassan, E.M. (2018) Effectiveness of poultry litter amendments on bacterial survival and Eimeria oocyst sporulation. Vet. World,
11(8): 1064-1073.

14. Herigstad, B., Hamilton, M. and Heersink, J. (2001) How to optimize the drop plate method for enumerating bacteria. $J$. Microbiol. Methods, 44(2): 121-129.

15. Soliman, E.S., Moawed, S.A. and Ziaan, A.M.G. (2016) Assessing cleaning and disinfection regime in a slaughterhouse against carcasses contamination. Adv. Anim. Vet. Sci., 4(9): 449-457.

16. Soliman, E.S. and Hassan, R.A. (2020) Influence of housing floor on air quality, growth traits, and immunity in broiler chicken farms. Adv. Anim. Vet. Sci., 8(9): 997-1008.

17. Soliman, E.S. and Hassan, R.A. (2019) Impact of lighting color and duration on productive performance and Newcastle disease vaccination efficiency in broiler chickens. Vet. World, 12(7): 1052-1059.

18. Soliman, E.S. and Abdallah, M.S. (2020) Assessment of biosecurity measures in broiler's farms in the Suez Canal area Egypt using a seasonal prevalence of Salmonellosis. Vet. World, 13(4): 622-632.

19. National Research Council. (1994) Nutrient Requirements for Poultry. $9^{\text {th }}$ ed. National Research Council, New York.

20. Applegate, T.J. and Angel, R. (2014) Nutrient requirements of poultry publication: History and need for an update. $J$. Appl. Poult. Res., 23(3): 567-575.

21. Jana A. and Mondal A. (2013) Serotyping, pathogenicity and antibiogram of Escherichia coli isolated from raw poultry meat in West Bengal, India. Vet. Ital., 49(4): 361-365.

22. Thrusfield, M. (2005) Veterinary Epidemiology. $3^{\text {rd }}$ ed. Wiley Blackwell, Oxford. p228-330.

23. Soliman, E.S. and Hassan, R.A. (2017) Evaluation of superphosphate and meta-bisulfide efficiency in litter treatment on productive performance and immunity of broilers exposed to ammonia stress. Adv. Anim. Vet. Sci., 5(6): 253-259.

24. Kim, S.K. and Lee, J.H. (2016) Biofilm modeling systems. Korean J. Microbiol., 52(2): 125-139.

25. American Public Health Association, American Water Works Association, Water Environment Federation. (2012) Standard Methods for the Examination of Water and Wastewater. 22 ${ }^{\text {th }}$ ed. American Water Work Association Publications, Washington, DC.

26. American Public Health Association, American Water Works Association, Water Environment Federation. (2017) In: Rice, E.W., Baird, R.B. and Eaton, A.D., editors. Standard Methods for the Examination of Water and Wastewater. $23^{\text {rd }}$ ed. American Water Work Association Publications, Washington, DC.

27. Murray, P.R., Rosenthal, K.S. and Pfaller, M.A. (2015) Medical Microbiology. $8^{\text {th }}$ ed. Elsevier Health Sciences, Philadelphia, PA, USA.

28. Bancroff, J., Stevenes, A. and Turner, D. (1990) Theory and Practice of Histological Techniques. $3^{\text {rd }}$ ed. Churchill Livingston, Edinburgh, London.

29. Jones, M.L., Bancroft, J.D. and Gamble, M. (2008) Connective tissues and stains. In: Bancroft, J.D. and Gamble, M., editors. Theory and Practice of Histological Techniques. Churchill Livingstone Elsevier, Philadelphia, PA. p135-160.

30. Green, S.B. and Salking, N.J. (2016) Using SPSS for Windows and Macintosh, Books la Carte. $8^{\text {th }}$ ed. Pearson, London, United Kingdom.

31. Song, J., Xiao, K., Ke, Y.L., Jiao, L.F., Hu, C.H., Diao, Q.Y., Shi, B. and Zou, X.T. (2014) Effect of a probiotic mixture on intestinal microflora, morphology, and barrier integrity of broilers subjected to heat stress. Poult. Sci., 93(3): 581-588.

32. Mingmongkolchai, S. and Panbangred, W. (2018) Bacillus probiotics: An alternative to antibiotics for livestock production. J. Appl. Microbiol., 124(6): 1334-1346.

33. Mohammed, A.A., Jacobs, J.A., Murugesan, G.R. and Cheng, H.W. (2018) Effect of dietary synbiotic supplement on behavioral patterns and growth performance of 
broiler chickens reared under heat stress. Poult. Sci., 97(4): 1101-1108.

34. Wang, W.C., Yan, F.F., Hu, J.Y., Amen, O.A. and Cheng, H.W. (2018) Supplementation of Bacillus subtilis-based probiotic reduces heat stress-related behaviors and inflammatory response in broiler chickens. J. Anim. Sci., 96(5): 1654-1666.

35. Harrington, D., Sims, M. and Kehlet, A.B. (2016) Effect of Bacillus subtilis supplementation in low energy diets on broiler performance. J. Appl. Poult. Res., 25(1): 29-39.

36. Gadde, U., Oh, S.T., Lee, Y.S., Davis, E., Zimmerman, N., Rehberger, T. and Lillehoj, H.S. (2017a) The effects of direct-fed microbial supplementation, as an alternative to antibiotics, on growth performance, intestinal immune status, and epithelial barrier gene expression in broiler chickens. Probiotics Antimicrob. Proteins, 9(4): 397-405.

37. Gadde, U.D., Oh, S., Lee, Y., Davis, E., Zimmerman, N., Rehberger, T. and Lillehoj, H.S. (2017b) Dietary Bacillus subtilis-based direct-fed microbials alleviate LPS-induced intestinal immunological stress and improve intestinal barrier gene expression in commercial broiler chickens. Res. Vet. Sci., 114(1): 236-243.

38. Wang, H., Ni, X., Qing, X., Zeng, D., Luo, M., Liu, L. and Jing, B. (2017) Live probiotic Lactobacillus johnsonii BS15 promotes growth performance and lowers fat deposition by improving lipid metabolism, intestinal development, and gut microflora in broilers. Front. Microbiol., 8(1): 1073.

39. Ramlucken, U., Ramchuran, S.O., Moonsamy, G., Lalloo, R., Thantsha, M.S. and van Rensburg, C.J. (2020) A novel Bacillus based multi-strain probiotic improves growth performance and intestinal properties of Clostridium perfringens challenged broilers. Poult. Sci., 99(1): 331-341.

40. Abdelqader, A., Abuajamieh, M., Hayajneh, F. and Al-Fataftah, A.R. (2020) Probiotic bacteria maintain normal growth mechanisms of heat stressed broiler chickens. J. Therm. Biol., 92(1): 102654

41. Oh, J.K., Pajarillo, E.A.B., Chae, J.P., Kim, I.H., Yang, D.S. and Kang, D.K. (2017) Effects of Bacillus subtilis CSL2 on the composition and functional diversity of the faecal microbiota of broiler chickens challenged with Salmonella Gallinarum. J. Anim. Sci. Biotechnol., 8(5 January 2017): Article 1.

42. Ebrahimi, H., Rahimi, S., Khaki, P., Grimes, J.L. and Kathariou, S. (2016) The effects of probiotics, organic acid, and a medicinal plant on the immune system and gastrointestinal microflora in broilers challenged with Campylobacter jejuni. Turk. J. Vet. Anim. Sci., 40(3): 329-336.

43. Nishiyama, K., Seto, Y., Yoshioka, K., Kakuda, T., Takai, S., Yamamoto, Y. and Mukai, T. (2014) Lactobacillus gasseri SBT2055 reduces infection by and colonization of Campylobacter jejuni. PLoS One, 9(9): e108827.

44. Saint-Cyr, M.J., Guyard-Nicodème, M., Messaoudi, S., Chemaly, M., Cappelier, J.M., Dousset, X. and Haddad, N. (2016) Recent advances in screening of anti-Campylobacter activity in probiotics for use in poultry. Front. Microbiol., 7(31 May 2016): Article 553.

45. Carter, A., Adams, M., La Ragione, R.M. and Woodward, M.J. (2017) Colonisation of poultry by Salmonella Enteritidis S1400 is reduced by combined administration of Lactobacillus salivarius 59 and Enterococcus faecium PXN-33. Vet. Microbiol., 199(1): 100-107.

46. Neveling, D.P., van Emmenes, L., Ahire, J.J., Pieterse, E., Smith, C. and Dicks, L.M.T. (2019) Effect of a multi-species probiotic on the colonisation of Salmonella in broilers. Probiotics Antimicrob. Proteins, 12(3): 896-905.

47. Zhang, L., Ma, Q., Ma, S., Zhang, J., Jia, R., Ji, C. and Zhao, L. (2016) Ameliorating effects of Bacillus subtilis ANSB060 on growth performance, antioxidant functions, and aflatoxin residues in ducks fed diets contaminated with aflatoxins. Toxins, 9(1): 1-11.

48. Fan, Y., Zhao, L., Ma, Q., Li, X., Shi, H., Zhou, T., Zhang,
J. and Ji, C. (2013) Effects of Bacillus subtilis ANSB060 on growth performance, meat quality and aflatoxin residues in broilers fed moldy peanut meal naturally contaminated with aflatoxins. Food Chem. Toxicol., 59(1): 748-753.

49. Abdolmaleki, M., Saki, A.A. and Alikhani, M.Y. (2019) Protective effects of Bacillus sp. MBIA2.40 and Gallipro on growth performance, immune status, gut morphology and serum biochemistry of broiler chickens feeding by aflatoxin B1. Poult. Sci. J., 7(2): 185-194.

50. He, T.F., Long, S.F., Mahfuz, S., Wu, D., Wang, X., Wei, X.M. and Piao, X.S. (2019) Effects of probiotics as antibiotics substitutes on growth performance, serum biochemical parameters, intestinal morphology, and barrier function of broilers. Animals, 9(11): 985.

51. Hosseini, N.G., Modarressi, M.H., Mousavi, S.N. and Ebrahimi, M.T. (2019) Effects of indigenous spore-forming probiotic as feed supplement on performance and safety in broilers. J. Hell. Vet. Med. Soc., 70(4): 1841-1850.

52. Sobczak, A. and Kozłowski, K. (2015) The effect of a probiotic preparation containing Bacillus subtilis a Tcc pTa-6737 on egg production and physiological parameters of laying hens. Ann. Anim. Sci., 15(3): 711-723.

53. Kim, W.H. and Lillehoj, H.S. (2019) Immunity, immunomodulation, and antibiotic alternatives to maximize the genetic potential of poultry for growth and disease response. Anim. Feed Sci. Technol., 250(1): 41-50.

54. Ribeiro, V. Jr., Albino, L.F.T., Rostagno, H.S., Barreto, S.L.T., Hannas, M.I., Harrington, D., Dearaujo, F.A., Ferrei Raj, R.H.C. and Ferreira, M.A. (2014) Effects of the dietary supplementation of Bacillus subtilis levels on performance, egg quality and excreta moisture of layers. Anim. Feed Sci. Technol., 195(1): 142-146.

55. Buta, A., Oprea, O., Sevciuc, P., Daradics, Z., Spatariu, S. and Ognean, L. (2020) Analysis of the influence of a nutraceutical supplement with probiotic effects on health index and productive performance in broiler chicken. Agrolife Sci. J., 9(1): 54-63.

56. Meyer, M.M., Fries-Craft, K.A. and Bobeck, E.A. (2020) Composition and inclusion of probiotics in broiler diets alter intestinal permeability and spleen immune cell profiles without negatively affecting performance. J. Anim. Sci., 98(1): skz383.

57. Dong, Y.X., Li, R., Liu, Y., Ma, L.Y., Zha, J.H., Qiao, X.B., Chai T.J. and $\mathrm{Wu}, \mathrm{B}$. (2020) Benefit of dietary supplementation with Bacillus subtilis BYS2 on growth performance, immune response, and disease resistance of broilers. Probiotics Antimicrob. Proteins, 12(4): 1385-1397.

58. Hrnčár, C., Gašparovič, M., Weis, J., Arpášová, H., Pistová, V., Fik, M. and Bujko, J. (2016) Effect of threestrain probiotic on productive performance and carcass characteristics of broiler chickens. Sci. Papers Anim. Sci. Biotechnol., 49(2): 149-154.

59. Hassan, H.M.A., Samy, A., Youssef, A.W. and Mohamed, M.A. (2018) Using different feed additives as alternative to antibiotic growth promoter to improve growth performance and carcass traits of broilers. Int. J. Poult. Sci., 17(6): 255-261.

60. Javandel, F., Nosrati, M., van den Hoven, R., Seidavi, A., Laudadio, V. and Tufarelli, V. (2019) Effects of Hogweed (Heracleum persicum) powder, flavophospholipol, and probiotics as feed supplements on the performance, carcass and blood characteristics, intestinal microflora, and immune response in broilers. J. Poult. Sci., 56(4): 262-269.

61. Alagawany, M., Abd El-Hack, M.E., Farag, M.R., Sachan, S., Karthik, K. and Dhama, K. (2018) The use of probiotics as eco-friendly alternatives for antibiotics in poultry nutrition. Environ. Sci. Pollut. Res., 25(11): 10611-10618.

62. Harimurti, S. and Ariyadi, B. (2015) Effect of indigenous probiotics lactic acid bacteria on the small intestine histology structure and the expression of mucins in the ileum of broiler chickens. Int. J. Poult. Sci., 14(5): 276-278. 
63. Trani, A., Loizzo, P., Calvano, C.D., Cassone, A., Gambacorta, G., Zambrini, A.V., Zambonin, C.G. and Faccia, M. (2016) Effects of a Lactobacillus acidophilus D2 enriched diet on yolk protein in hen eggs. Eur. Poult. Sci., 80(2016): Article 124.

64. Yisa, T.A., Ibrahim, O.A., Tasdu, S.M. and Yakubu, U.P. (2015) Effect of probiotics (Lactobacillus acidophilus and Bifidobacterium bifidum) as immune stimulant on hybrid Catfish heteroclarias. Br. Microbiol. Res. J., 9(1): 1-6.

65. Awais, M.M., Jamal, M.A., Akhtar, M., Hameed, M.R., Anwar, M.I. and Ullah, M.I. (2019) Immunomodulatory and ameliorative effects of Lactobacillus and Saccharomyces based probiotics on pathological effects of eimeriasis in broilers. Microb. Pathog., 126(1): 101-108.

66. Ashraf, R. and Shah, N.P. (2014) Immune system stimulation by probiotics microorganisms. Crit. Rev. Food Sci. Nutr., 54(7): 938-956.

67. Gonmei, G., Sapcota, D., Saikia, G.K., Deka, P., Mahanta, J.D., Kalita, N., Saikia, B.N. and Talukdar, J.K. (2019) Studies on immune response to Newcastle disease virus in broiler chickens fed with Lactobacillus reuteri PIA16 isolated from the gut of indigenous chicken of Assam, India. Vet. World, 12(8): 1251-1255.

68. Sarwar, N., Mehmood, A., Sheraz, A. and Noman, M. (2019) Protective effect of probiotics in combination with vaccination on antibody response, biochemical and hematological indices in broilers. Pak. Vet. J., 39(3): 451-454.

69. Shankar, P.A., Premavalli, K., Omprakash, A.V., Kirubakaran, J.J., Hudson, G.H. and Vairamuthu, S. (2018) Effect of dietary yeast supplementation on serum biochemical profile of broiler chicken. Indian Vet. J., 95(6): 13-15.

70. Deraz, S.F. (2018) Synergetic effects of multispecies probiotic supplementation on certain blood parameters and serum biochemical profile of broiler chickens. J. Anim.
Health Prod., 6(1): 27-34.

71. Hussein, E. and Selim, S. (2018) Efficacy of yeast and multi-strain probiotic alone or in combination on growth performance, carcass traits, blood biochemical constituents, and meat quality of broiler chickens. Livest. Sci., 216(1): 153-159.

72. Hussein, E.O.S., Ahmed, S.H., Abudabos, A.M., Aljumaah, M.R., Alkhlulaifi, M.M., Nassan, M.A., Suliman, G.M., Naiel, M.A.E. and Swelum, A.A. (2020) Effect of antibiotic, phytobiotic and probiotic supplementation on growth, blood indices and intestine health in broiler chicks challenged with Clostridium perfringens. Animals, 10(3): 507.

73. Abramowicz, K., Krauze, M. and Ognik, K. (2020) Use of Bacillus subtilis PB6 enriched with choline to improve growth performance, immune status, histological parameters and intestinal microbiota of broiler chickens. Anim. Prod. Sci., 60(5): 625-634.

74. Adhikari, P., Kiess, A., Adhikari R. and Jha, R. (2020) An approach to alternative strategies to control avian coccidiosis and necrotic enteritis. J. Appl. Poult. Res., 29(2): 515-534.

75. Huang, T., Peng, X.Y., Gao, B., Wei, Q.L., Xiang, R., Yuan, M.G. and Xu, Z.H. (2019) The effect of Clostridium butyricum on gut microbiota, immune response and intestinal barrier function during the development of necrotic enteritis in chickens. Front. Microbiol., 10(11 October 2019): Article 2309.

76. Olnood, C.G., Beski, S.S., Choct, M. and Iji, P.A. (2015) Novel probiotics: Their effects on growth performance, gut development, microbial community and activity of broiler chickens. Anim. Nutr., 1(3): 184-191

77. Kogut, M.H. (2019) The effect of microbiome modulation on the intestinal health of poultry. Anim. Feed Sci. Technol., 250(1): 32-40. 